\title{
Efficient deadline-aware scheduling for the analysis of Big Data streams in public Cloud
}

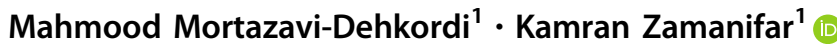

Received: 8 February 2018/Revised: 20 November 2018 / Accepted: 8 January 2019/Published online: 14 February 2019

(C) The Author(s) 2019

\begin{abstract}
The emergence of Big Data has had a profound impact on how data are analyzed. Open source distributed stream processing platforms have gained popularity for analyzing streaming Big Data as they provide low latency required for streaming Big Data applications using Cloud resources. However, existing resource schedulers are still lacking the efficiency and deadline meeting that Big Data analytical applications require. Recent works have already considered streaming Big Data characteristics to improve the efficiency and the likelihood of deadline meeting for scheduling in the platforms. Nevertheless, they have not taken into account the specific attributes of analytical application, public Cloud utilization cost and delays caused by performance degradation of leasing public Cloud resources. This study, therefore, presents BCframework, an efficient deadline-aware scheduling framework used by streaming Big Data analysis applications based on public Cloud resources. BCframework proposes a scheduling model which considers public Cloud utilization cost, performance variation, deadline meeting and latency reduction requirements of streaming Big Data analytical applications. Furthermore, it introduces two operator scheduling algorithms based on both a novel partitioning algorithm and an operator replication method. BCframework is highly adaptable to the fluctuation of streaming Big Data and the performance degradation of public Cloud resources. Experiments with the benchmark and real-world queries show that BCframework can significantly reduce the latency and utilization cost and also minimize deadline violations and provisioned virtual machine instances.
\end{abstract}

Keywords Streaming Big Data analysis query · Deadline-aware scheduling · Cloud-based stream processing

\section{Introduction}

The emergence of data-intensive applications has rapidly increased the volume, variety and velocity of the generated data during its lifecycle which represents a major challenge for many organizations and is known as the Big Data problem [1]. Streaming Big Data is related to the velocity dimension of Big Data and refers both to how fast data are generated and how fast they need to be analyzed [2]. Analysis of streaming Big Data is the last and most important stage of the streaming Big Data lifecycle in

Kamran Zamanifar

zamanifar@eng.ui.ac.ir

Mahmood Mortazavi-Dehkordi

mortazavi@shbu.ac.ir

1 Software Department, Computer Engineering Faculty, University of Isfahan, Isfahan, Iran analytical applications [3]. The real-world examples of the applications can be in the form of analytical queries to analyze the network traffic, healthcare data, financial transactions or web-clicks [4]. As the structure of the queries is usually complex and the input streams of the queries have high intensity of data, they can benefit from large-scale infrastructures [5].

Among such infrastructures, public Cloud is an appropriate infrastructure to host the queries because it can operate as pay-per-use model and is able to provide dynamic resource scaling in response to the fluctuating resource demand of the queries. This Cloud utilization model is called Infrastructure as a Service (IaaS) and can be used by the Cloud-based stream computing platforms to schedule accepted soft deadline-constrained queries using the leased resources. For a soft deadline-constrained query, the maximal value of the computation is not achieved if the assigned deadline is violated and additional deadline 
violations can occur when the required resources have been leased from public Cloud. As reported by [6], public Cloud does not provide regular performance in terms of execution and data transfer time. The performance fluctuation of public Cloud resources can vary up to $30 \%$ for execution and up to $65 \%$ for data transfer. The performance fluctuation can lead to delayed execution of the query operators and if the operators be part of the query critical path, a deadline violation can occur.

The efficient scheduling of the provisioned Cloud resources can increase the likelihood of the deadline meeting and reduce the average tuple latency and utilization cost of queries accepted by the distributed stream computing platforms. However, current platforms do not adequately address the problem of efficient scheduling of soft deadline-constrained streaming Big Data analytic queries in public Cloud. Recent works have proposed solutions to reduce the average tuple latency of streaming Big Data queries in private clouds. However, to the best of our knowledge, the soft-deadline meeting of the analytical queries, the reduction of the public Cloud utilization cost of the analytical queries and performance variation of public Cloud resources are not jointly investigated.

To address these limitations, we developed a scheduler, BCframework, with low tuple latency and utilization cost suitable for soft deadline-constrained streaming Big Data analytic queries by considering the poor performance of public Cloud resources. Our approach is inspired by the idea that using the structure of soft deadline-constrained streaming Big Data analytic query to partition the query critical path operators and applying a distinct scheduling strategy for each partition can reduce both the average tuple latency and the utilization cost of the soft deadlineconstrained query. More precisely, as a sample query shown in Fig. 1, the weight of the outgoing edge of the operator $\mathrm{O}_{\mathrm{Join}}\left(\mathrm{J}_{1}\right)$ is considerably higher than the operator $\mathrm{O}_{\text {Project }}\left(\Pi_{2}\right)$ outgoing edge; using this edge weight difference can result in multi partitions of operators and therefore, a specific scheduling strategy can be applied for each partition. Furthermore, we can replicate the query operators and schedule them using the specific scheduling strategy to the provisioned resource to mitigate the performance violation of public Cloud resources.
Key contributions of this study can be summarized as follows:

- Formal definition of scheduling model for the deadlineconstrained Big Data stream analytic query in public Cloud.

- Automated provisioning of the public Cloud resources required by a deadline-constrained streaming Big Data analytic query.

- Scheduling of the deadline-constrained streaming Big Data analytic query operators in public Cloud resources.

- Automated replication and scheduling of the streaming Big Data analytic query replicated operators to the provisioned public Cloud resources.

- Simulation and performance evaluation of BCframework.

The remainder of this paper is organized as follows. Section 2 reviews the related works on Big Data and Cloudbased stream processing schedulers. In Sect. 3, the formal definition of the scheduling model for the deadline-constrained Big Data stream analytic query in public Cloud is presented. Section 4 presents the conceptual architecture of BCframework. Furthermore, it focuses on the partitioning, provisioning, scheduling and replication algorithms of BCframework. Section 5 shows the experimental environment, parameters setup, and the experimental results of BCframework. Finally, conclusions and future works are given in Sect. 6.

\section{Related works}

This section presents two broad categories of related works: scheduling and provisioning in cloud-based distributed stream processing (DSP) open source platforms and the scheduling and provisioning for stream Big Data queries or tasks.
Fig. $1 B q_{i j}$, a sample soft deadline-constrained streaming Big Data analytic query instance

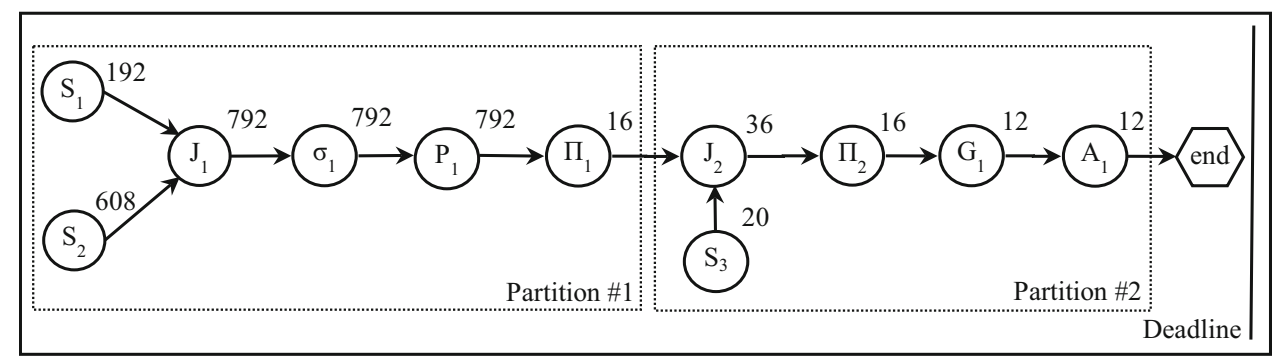




\subsection{Cloud-based DSP scheduling}

In this section, BCframework as a DSP scheduler is compared with the recent state-of-the-art studies in terms of the proposed task, resource and scheduling models.

$\mathrm{Xu}$ et al. designed and implemented a stream processing system developed based on Storm platform [7] whose traffic-aware scheduler assigns and reassigns accepted tasks at runtime [8]. Furthermore, the scheduler aims to minimize the inter-node and inter-process traffic loads and also monitors the overload of machines. In comparison with BCframework, this study is neither resource-aware nor task-aware scheduler because its task model only considers the tasks as a set of given threads and models resource as a set of given processes. Furthermore, its scheduling model only takes into account the inter-node traffic optimization.

In [9], Storm platform is extended to operate in geographically distributed and highly variable environments. Authors present a distributed scheduler which is QoSaware and operates at runtime. It takes into account the latency, resource utilization and resource availability as QoS attributes. The scheduling model aims to solve two placement problems: assignment of the task operators to virtual machines and assignment of the virtual machines to the physical machines where in comparison with our work, BCframework only considers the operator to virtual machine assignment. Furthermore, its scheduler can be distributed among multiple worker nodes which BCframework is not capable of. In addition, no special strategies are proposed by the authors while BCframework has proposed efficient strategies for virtual machine assignment. And also, our task model models tasks as a set of the operators which is modelled as directed graph.

Li et al. proposed a scheduling framework which predicts the processing time of the application based on the structure of the task [10]. They also present a scheduling algorithm which assigns threads to machines based on the prediction result. In comparison with BCframework, its task model considers tasks as a set of threads and processes; its resource model considers resources as a set of machines, however their load and capacity is not considered. Furthermore, its only objective is to minimize the inter-operator traffic load of a task.

In $[11,12]$, the placement problem for DSP is studied and a scheduler for Storm platform is presented based on Integer Linear Programming. Similar to BCframework, they model tasks as a graph. Their resource model considers machines with respect to QoS metrics like resource availability. Furthermore, their scheduling objectives model the two QoS metrics for resource availability and response time. In addition, their solutions take into account the network delay while the capacity of the machines is not considered.

De Matteis et al. aim to provision resources needed for data stream applications in the presence of workload fluctuation [13]. They use a control-theoretic method to find an optimal stream application configuration to address latency constraints. In comparison with BCframework, they mainly focus on the resource provisioning problem where the focus of BCframework is on both resource provisioning and scheduling problems.

The Flink platform [14] includes a scheduling framework which is able to schedule a path of operators of an accepted task or multiple paths of multiple tasks in a machine. As the framework does not take into account the QoS requirements, its scheduler is not QoS-aware. In [14], a scheduler for Flink, S-Flink, is proposed using SDN controllers to place tasks to the underlying resources while considering the QoS requirements of the tasks. In comparison with BCframework, the resource model of S-Flink is defined based on software defined network infrastructures while BCframework's resource model relies on traditional network infrastructures. In [15], Heron, a new distributed system for managing stream processing application, is developed to overcome the limitations of Storm. In this system, Aurora [16] is used as a generic resource scheduling framework where the specific definition of scheduling and provisioning strategies is done by the users of Aurora while BCframework already includes predefined scheduling and provisioning strategies.

In [17] authors propose a cloud-based scalable and elastic stream processing engine which is able to process large data stream volumes using a novel parallelization technique. The engine aims to minimize the computational resources used while balancing the traffic load. In comparison with BCframework, they mainly focus on the query parallelization while the focus of BCframework is on the scheduling of queries which are already paralleled.

Li et al. proposed a task optimization and scheduling algorithms which their scheduling algorithm takes into account the structure and traffic load of task and resources characteristics [18]. The task model is a Directed Acyclic Graph (DAG) and the scheduling algorithm assigns operators to machines. The objective of algorithms is to minimize the inter-node network traffic. In comparison with BCframework, their task, resource and scheduling models have close assumption with BCframework but their algorithms are not specially designed for analytic queries and their solution has some limitations.

In summary, most optimization strategies for improving the cloud-based DSP scheduling and provisioning consider the task structure and inter-machine traffic or machine load aspects for their algorithms. However, our proposed algorithms could improve DSP scheduling and provisioning by 
jointly considering the task structure, inter-machine network traffic and workload of machines while taking into account the public Cloud resources characteristics.

\subsection{Streaming big data scheduling}

In this section, BCframework as a streaming Big Data scheduler is compared with the recent state-of-the-art studies in terms of the proposed task, resource and scheduling models.

Sun et al. proposed a real-time scheduling framework which aims to meet the low response time and high energy efficiency of the query [19]. Similar to BCframework, it models tasks as graph which encompasses operators and their inputs and outputs. Furthermore, it models resources by considering resource capacity and the aim of its scheduling model is to minimize response time and maximize the energy efficiency of the machines. However, the variety dimension of streaming Big Data is not considered.

In [20], a fault tolerance framework is presented which allocates tasks to fault tolerant machines before the execution of tasks and also reassigns the tasks to the machines with lower response time. The task model considers task as graph and its resource model is similar to BCframework and models the capacity of the resources. Its scheduling model aims to minimize the response time and maximize the system reliability where in comparison with BCframework the $3 \mathrm{Vs}$ model of streaming Big Data is not considered completely.

Kaur et al. predict the data characteristics (5Vs) of streaming Big Data and are expressed in CoBA value which is used to determine the required machines of the tasks [21]. In comparison with BCframework, It just considers the streaming Big Data and there is no model for task and resources.

In [22], a stable scheduling is presented which allocates tasks to the stable machines before the execution of tasks and reassigns the task to the machines with lower response time. Similar to BCframework, it models tasks as graph which encompasses operators with their input and outputs and the capacity is included in the resource model. Its scheduling model aims to minimize the response time and maximize the stability of the machines. However, the $3 \mathrm{Vs}$ model of Big Data is not considered completely.

Kaur et al. estimated data characteristics of streaming Big Data based on the $4 \mathrm{Vs}$ model as a value named Characteristics of Data (CoD) [23]. Furthermore, Self-Organizing Maps (SOM) is used to allocate cloud resources to streaming Big Data using its estimated CoD. In comparison with BCframework, this work only considers the streaming Big Data, the streaming Big Data queries are not considered.
In [24], authors derived effective optimization rules which are used to reconfigure the structure of an accepted task at runtime using real-time performance statistics. In comparison with BCframework, the result of this work can be used as an optimized input query to BCframework.

Tolosana-Calasanz et al. developed a system architecture which accepts concurrent data streams and are able to regulate and control the received streams. Furthermore, their architecture provisions the required resources for processing the streams while maximizing the Cloud provider revenue [25]. In comparison with BCframework, their work does not take into account the query model and scheduling model for Big Data stream.

In [26], authors propose a static hybrid provisioning mechanism which prioritizes and manages the grouped VM requests to determine the required VM instances. In comparison with BCframework, their mechanism solely focuses on managing VM requests while there are no query, data or scheduling models for Big Data stream.

Zhang et al. propose a cloud scheduling model to overcome the speed and size difficulties of streaming Big Data analysis based on Markov chain prediction model [27]. In comparison with BCframework, the authors propose the data model for streaming Big Data analysis but the query model is not considered.

In [28] authors propose a VM allocation and placement strategy which consider the type of application and the capacity and dynamic load of the physical machines to assign them VM instances. In comparison with BCframework, there is no data or query model proposed in their work.

Baughman et al. propose a novel forecasting and predictive model which can be used for the analysis of Big Data streams to cover the velocity dimension of Big Data stream [29]. In comparison with BCframework, there is no data, query and scheduling model proposed in their research.

In [30] an elastic online scheduling framework is proposed which encompasses features such as online scheduling of Big Data stream applications, profiling the mathematical relationship between system response time, multiple application fairness and high-velocity continuous stream. In comparison with BCframework, its resource model does not take into account the analytical characteristics of the streaming Big Data queries.

In summary, the current optimization strategies for improving the scheduling and provisioning of streaming Big Data only consider some aspects of the Big Data characteristics. However, BCframework's scheduling and provisioning algorithms which not only considers all of the mentioned aspects but it is also suited for analytical queries of streaming Big Data. 


\section{Problem statement}

In the context of stream Big Data computing, a set of deadline-constrained streaming Big Data analytic queries is hosted by a public Cloud provider and one or multiple streams of Big Data flow continuously through the instances of the accepted queries.

Efficient execution of each query (instance) is mainly achieved by the efficient provisioning and scheduling decisions made about public Cloud resources. To incorporate these factors into the provisioning and scheduling decisions, we bring some related definitions below using notations addressed in Table 1.

\subsection{Data model}

In BCframework, a data stream $D S$ is a sequence of $d s$ data sets, where each data set $D s_{q}$ is a set of $q l$ number of $t_{q r}$ tuple. A continuous data stream is an infinite sequence of data sets, and parallel continuous data streams are multiple streams that can be processed at the same time. Big data stream $B s$ is a continuous data stream with high velocity, high volume and a wide variety of data sets. We applied the structured, semi-structured and unstructured definitions of Big Data [1] to formally define $B s$ using Definition 1.

Definition 1 (Big Data stream) The Big Data stream $B s$ is a continuous stream. The volume of its data sets is defined by $B s^{V o}$, velocity of its data sets is defined by $B s^{V e}$ and the variety of its data sets is defined by $B s^{V a}$, where data type of each $B s^{V a}$ can be structured, semi-structured or unstructured. In addition, let $B f$, Big Data flow, be defined as edge flows $B s$.

\subsection{Query model}

In BCframework, a deadline-constrained Big Data stream analytic query can be defined as an aperiodic continuous query with a set of operators which analyzes one or multiple input Big Data streams $B s(s)$ and produces most of its output Big Data streams $B s(s)$ within associated soft deadline. We formally define the query using Definition 2.

Definition 2 (Deadline-constrained Big Data stream analytic query) $B q_{i j}$ can be expressed as a direct acyclic graph and characterized by four-tuple $B q_{i j}=\left(B q_{i}^{O}, B q_{i}^{E}, B q_{i j}^{D L}, B q_{i}^{W}\right)$. The operator $O_{t}$ of $B q_{i}$ is characterized by the triplet $O_{t}=\left(O_{t}^{I n p}, O_{t}^{\text {out }}, O_{t}^{\text {tpe }}\right)$. The in-degree of operator $O_{t}$ is the number of incoming edges, and the out-degree of operator $O_{t}$ is the number of the outgoing edges. The source operator is the operator whose in-degree is zero, and the end operator is the operator whose out-degree is zero. A $B q_{i}$ has at least one source and one end operator.

Figure 1 shows the $j$-th execution instance of the deadline-constrained Big Data stream analytic query $B q_{i}$ whose $B q_{i}^{O}$ includes 12 operators and a soft deadline $B q_{i j}^{D L}$ is associated to $B q_{i j}$. Source operators are $O_{S 1}, O_{S 2}, O_{S 3}$ and the end operator is $O_{\text {end }}$. The operator $O_{J 1}$ has two edges $E_{S 1 J 1}$ and $E_{S 2 J 1}$ where $E_{S 1 J 1}$ flows Big Data flow $B f$ as input Big Data stream $B s, O_{J 1}^{I n p}$, and $E_{S 2 J 1}$ flows another $O_{J 1}^{I n p}$. The volume of $B s, B s^{V o}$, of the $E_{S 1 J 1}$ is estimated to be 192 bytes while its recently aggregated tuples $B q_{i}^{W}$ are not considered. As the $O_{J 1}$ is defined by user, its type $O_{J 1}^{t p e}$ is considered to be an original operator and it is not replicated by BCframework. $B q_{i j}$ is partitioned into two partitions Partition\#1 and Partition\#2 based on the output $B f$ of an operator $O_{t}^{\text {out }}$. This enables BCframework to apply different provisioning and scheduling strategies for each partition.

\subsection{Scheduling model}

In BCframework, the scheduler accepts a set of independent queries, provisions their required public Cloud resources and schedules all operators of the accepted queries on the provisioned resources to achieve the lowest average query latency and Cloud resource utilization cost with subject to the soft deadline constraint associated with the query instances. The following model reflects these factors.

Let $B Q$ denote a set of independent $b q$ queries accepted by the scheduler $B Q=\left\{B q_{1}, \ldots B q_{i}, \ldots, B q_{b q}\right\}$, Furthermore, let $V M$ denote a set of $v m$ virtual machines $V M=\left\{V m_{1}, \ldots, V m_{k}, \ldots, V m_{v m}\right\}$. Each $V m_{k}$ is charged per amount of time by public Cloud provider. In addition, for the execution of the query, there is no limit imposed on the number, type and provisioning time of $\mathrm{Vm}_{k}$.

The runtime matrix Runtime ${ }_{(b q \times o n) \times v m}^{j}$ indicates the estimated runtime of the $j$-th instance of all accepted queries operators Runtime $_{(b q \times o n) \times v m}^{j}=$ $\left(\begin{array}{cc}R_{11}^{j} & R_{b q \times o n \times 1}^{j} \\ R_{1 v m}^{j} & R_{b q \times o n \times v m}^{j}\end{array}\right)$, where $R_{t k}^{j}$ specifies the estimated runtime of the $j$-th instance of the operator $O_{t}$ on a VM type $V m_{k}$.

The schedule matrix Schedule ${ }_{(b q \times o n) \times v m}^{j}$ represents the assignment of the $j$-th instance of all operators of all accepted queries to the virtual machines Schedule $_{(b q \times o n) \times v m}^{j}=\left(\begin{array}{cc}S_{11}^{j} & S_{b q \times o n \times 1}^{j} \\ S_{1 v m}^{j} & S_{b q \times o n \times v m}^{j}\end{array}\right), \quad$ where $S_{t k}^{j}$ shows that the $j$-th instance of operator $O_{t}$ is assigned to the 
Table 1 The notation used in the problem statement

\begin{tabular}{|c|c|}
\hline Notion & Definition \\
\hline \multicolumn{2}{|c|}{ Data model } \\
\hline$D S$ & Data stream, $D S=\left\{D s_{1}, \ldots, D s_{q}, \ldots, D s_{d s}\right\}$ \\
\hline$D s_{q}$ & Data set, $D s_{q}=\left\{t_{q 1}, \ldots, t_{q r}, \ldots, t_{q l}\right\}$ \\
\hline$t_{q r}$ & $r$-th Tuple of $D s_{q}$ \\
\hline Bs & Big data stream \\
\hline$B s^{V o}$ & Volume of $B s, B s^{V o}=\left\{D s_{1}^{V o}, \ldots, D s_{q}^{V o}, \ldots, D s_{d s}^{V o}\right\}$ \\
\hline$B s^{V e}$ & Velocity of $B s, B s^{V e}=\left\{D s_{1}^{V e}, \ldots, D s_{q}^{V e}, \ldots, D s_{d s}^{V e}\right\}$ \\
\hline$B s^{V a}$ & Variety of $B s, B s^{V a}=\left\{D s_{1}^{V a}, \ldots, D s_{q}^{V a}, \ldots, D s_{d s}^{V a}\right\}$ \\
\hline$D s_{q}^{V o}$ & Volume of data set $D s_{q}$ (measured in byte) \\
\hline$D s_{q}^{V e}$ & Velocity of data set $D s_{q}$ (measured in byte/second) \\
\hline$D s_{q}^{V a}$ & Variety of data set $D s_{q}$ \\
\hline$B f$ & Big Data flow \\
\hline \multicolumn{2}{|c|}{ Query model } \\
\hline$B q_{i}$ & Deadline-constrained Big Data stream analytic query $i$ \\
\hline$B q_{i j}$ & Instance $j$ of a deadline-constrained Big Data stream analytic query $i, B q_{i j}=\left(B q_{i}^{O}, B q_{i}^{E}, B q_{i j}^{D L}, B q_{i}^{W}\right)$ \\
\hline$B q_{i}^{O}$ & Finite set of $o n$ number of $B q_{i}$ operators $O=\left\{O_{1}, \ldots, O_{t}, \ldots, O_{o n}\right\}$ \\
\hline$B q_{i}^{E}$ & Finite set of $B q_{i}$ directed edges $E=\left\{E_{11}, \ldots, E_{p q}, \ldots E_{\text {onon }}\right\}$ \\
\hline$B q_{i j}^{D L}$ & Soft deadline associated to the $j-t h$ instance of query $B q_{i}$ \\
\hline$B q_{i}^{W}$ & Window size of query $B q_{i}$ \\
\hline$E_{p q}$ & Execution precedence between two operators $p$ and $q$ which is member of $B f$ \\
\hline$O_{t}$ & Query Operator, $O_{t}=\left(O_{t}^{\operatorname{Inp}}, O_{t}^{\text {out }}, O_{t}^{\text {tpe }}\right)$ \\
\hline$O_{t}^{\operatorname{In} p}$ & Set of input Big Data flows $B f(s)$ to operator $O_{t}$ \\
\hline$O_{t}^{\text {out }}$ & Set of output Big Data flow $(\mathrm{s}) B f(s)$ from operator $O_{t}$ \\
\hline$O_{t}^{t p e}$ & Type of $O_{t}$ as an original or replicated operator \\
\hline \multicolumn{2}{|c|}{ Scheduling model } \\
\hline$B Q$ & Set of independent $b q$ queries accepted by the scheduler $B Q=\left\{B q_{1}, \ldots B q_{i}, \ldots, B q_{b q}\right\}$ \\
\hline$V M$ & Set of $v m$ virtual machines, $V M=\left\{V m_{1}, \ldots, V m_{k}, \ldots, V m_{v m}\right\}$ \\
\hline$V m_{k}$ & Virtual machine $k, V m_{k}=\left(V m_{k}^{C u}, V m_{k}^{R m}, V m_{k}^{C t}\right)$ \\
\hline$V m_{k}^{C u}$ & CPU capacity of $V m_{k}$ \\
\hline$V m_{k}^{R m}$ & Memory capacity of $V m_{k}$ \\
\hline$V m_{k}^{C t}$ & Utilization cost of $V m_{k}$ \\
\hline
\end{tabular}

virtual machine $V m_{k}$. To meet the soft deadline, we should meet the constraint defined by Eq. (1), so that the assignment of $j$-th instance of the end operator $S_{\text {endk }}^{j}$ to the any $V m_{k}$ would not exceed the deadline at which operators can be executed.

$S_{\text {endk }}^{j} \leq B q_{i j}^{D L} \quad \forall k \in\{1 \ldots v m\}$

In BCframework, the schedule model used by its scheduler is defined by Definition 3.

Definition 3 (scheduling model Sm) Sm is the scheduling model used in BCframework and is represented by four- tuple $\quad S m=\{B Q, V M, C O, O B\}, \quad$ where $\quad B Q=$ $\left\{B q_{1}, \ldots B q_{i}, \ldots, B q_{b q}\right\}$ is the set of $b q$ accepted queries and $V M$ is the set of provisioned virtual machines as the underlying public Cloud resources of BCframework. $C O$ is the constraint defined by Eq. (1) and $O B$ is the objective function of $\mathrm{Sm}$ which is defined to minimize the average tuple latency and the Cloud resource utilization cost of each $B q_{i j}$ instance of the accepted queries $B Q$ according to Eq. (2) 


$$
\begin{array}{r}
O B\left(B q_{i j}\right)=\operatorname{Min}\left(\operatorname{Avg}\left(\operatorname{Latency}\left(B q_{i j}\right)\right)\right. \\
\text { and } \left.\operatorname{Avg}\left(\operatorname{Cost}\left(B q_{i j}\right)\right)\right),
\end{array}
$$

where Latency $\left(B q_{i j}\right)$ represents the tuple latency of $B q_{i j}$ and it is defined according to Eq. (3)

$\operatorname{Latency}\left(B q_{i j}\right)=\operatorname{Time}\left(B q_{i j} \cdot O_{\text {end }}^{\text {Out }}\right)-\operatorname{Time}\left(B q_{i j} \cdot O_{\text {source }}^{\text {Inp }}\right)$,

where Time $\left(B q_{i j} . O_{\text {end }}^{\text {Out }}\right)$ is the timestamp of the earliest $B s$ of the output $B f$ in $B q_{i j}^{\prime} s$ end operator and Time $\left(B q_{i j} . O_{\text {source }}^{\text {Inp }}\right)$ is the timestamp of the earliest $B s$ of the $B q_{i j}^{\prime} s$ source operators. Furthermore, $\operatorname{Cost}\left(B q_{i j}\right)$ is defined by the Amazon AWS EC2 pricing model [31].

\section{BCframework}

The goal of BCframework is to minimize the average tuple latency and public Cloud resource utilization cost of the accepted queries and increases the likelihood of completing the execution of the accepted queries (instances) before or on their deadlines. To reach this goal, two related sub problems have to be solved: provisioning and scheduling. In BCframework, the provisioning problem consists of the determination on number and type of VM to be used for the query instance $B q_{i j}$ execution and the scheduling problem includes the determination of the placement and ordering of the $B q_{i j}$ operator instances on the provisioned VMs.

The BCframework's idea for solving the problems is to use more than one provisioning and scheduling strategy for the query $B q_{i}$. The rationale of this idea is related to an attribute of analytical queries where the output size of analytic query end operators is usually smaller than the input size of its source operators [32]. BCframework makes use of this characteristic to realize its idea by profiling the output size of $B q_{i}$ operators and partitioning the operators using the profiled output size.

To support the idea, the objectives of BCframework provisioning and scheduling algorithms are established based on the characteristics of the identified partitions. For the first partition, the objective of the algorithms is to minimize the average tuple latency of this partition operators where the objective of algorithms for the second partition is to minimize the public Cloud resource utilization cost of this partition operators. The algorithms apply their own provisioning and scheduling strategies to achieve the objectives of each partition.

To mitigate the delay affected by the poor performance of public Cloud resources, BCframework replicates a set of selected $B q_{i j}$ operators on the idle slots of the provisioned VMs. In addition, BCframework reacts to missed deadlines of previous executed query instances and incrementally provisions additional $\mathrm{Vm}$ instances to increase the deadline meeting probability.

Furthermore, BCframework adapts the algorithm presented in [33] which proposed a deadline and cost aware algorithms for provisioning and scheduling using critical path idea for applications which are developed using workflow paradigm and deployed on cloud environment. Our algorithms extend the critical path idea to develop deadline and cost aware scheduling and provisioning algorithms for Big Data stream analytic queries which are developed based on the dataflow paradigm. In addition, our algorithms take into account the performance degradation of public Cloud resources.

\subsection{BCframework algorithms}

This section presents the detail of the algorithms used in the BCframework. First, it is described the Bpartitioner algorithm, which is used to partition the selected query $B q_{i}$. $B q_{i}$ is selected among the accepted queries based on the EDF strategy [2] where prioritizes earliest deadline query first. Then the partial critical path (PCP) algorithm is described, which is applied to determine the critical paths of $B q_{i}$. Subsequently the Oscheduler and Rscheduler algorithms are described that are used for the provisioning and scheduling of $B q_{i}$ original and replicated operator respectively. Finally, Deadline Controller algorithm to decrease the deadline violation of the query instances is explained.

Figure 2 demonstrates the architecture of BCframework. Bpartitioner module takes two parameters Vm Capacity determined by the monitor module and Query $B q_{i}$ accepted by BCframework to implement the Bpartitioner algorithm and to annotate the partitioned query $B q_{i}^{\prime} . P C P$ module uses Load and Traffic of Vm received form monitor module to implement the Partial Critical Path algorithm and determines the $P C P$ of $B q_{i}^{\prime}$. Oscheduler and Rscheduler modules implement the Oscheduler and Rscheduler algorithms respectively where Oscheduler uses $P C P$ to generate schedule Schedule and Rscheduler uses Schedule to generate schedule with replicated operators Schedule'. Deadline Controller module receives the execution status of $B q_{i j}$ and implements the Deadline Controller algorithm to provision additional $\mathrm{Vm}$ instances.

\subsubsection{Query partitioning algorithm}

The aim of partitioning algorithm is to partition the operators of $B q_{i}$ in way that the different scheduling strategies can be applied for each partition. This algorithm uses the defined outBfsize metric to partition $B q_{i}$ into at most two 
Fig. 2 The conceptual architecture of BCframework

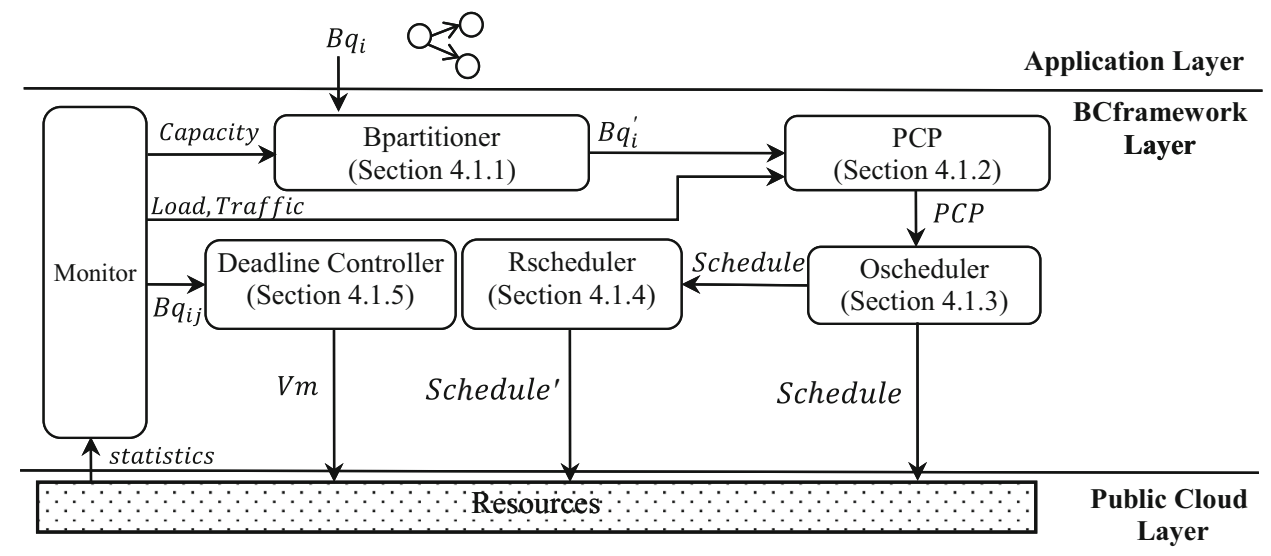

distinct operator partitions named $B q_{i}^{p 1}, B q_{i}^{p 2}$. The algorithm examines the query structure to estimate the value of a $B f$ metric named outBfsize, which indicates the estimated output $B f$ size of an operator. As a result, the partition $B q_{i}^{p 1}$ contains operators whose average size of outBfsize is greater than that outBfsize for the operators of partition $B q_{i}^{p 2}$. The value of outBfsize metric is estimated according to Eq. (4).

$$
\text { outBfsize }\left(O_{t}\right)=\sum_{\mathrm{p}=0}^{\text {out-degree }\left(O_{t}\right)} \sum_{\mathrm{q}=1}^{q=d s} \operatorname{Size}\left(O_{t}^{\text {out }}{ }_{p} \cdot \operatorname{Ds}_{q}^{V_{O}}\right)
$$

In Eq. (4) $\sum^{q=d s} \operatorname{Size}\left(O_{t}^{\text {out }}\right.$. $\left.\mathrm{Ds}_{q}^{\mathrm{Vo}}\right)$ denotes the sum of the size of the redent data sets of the $p-t h$ output $B f$ of $O_{t}$. The recent data sets are a set of $t_{q l}$ tuples of the data set $D s_{i}$ and a set of ds number of data sets DS. The ds is determined by the query window size $B q_{i}^{W}$ and $B s^{V o}$ includes DS. To estimate the value of $\mathrm{Ds}_{k}^{V o}$ initially, $B q_{i}$ is executed by setting the number of the input $B f$ tuples of $B q_{i}$ to 1 and deploying on a provisioned $V m$ instance. Algorithm 1 describes how this metric is used to partition the $B q_{i}$ operators.

The algorithm operates in two main steps:

- Lines 1-4: Calculates the outBfsize metric of the source and end operators of $B q_{i}$. As there may be more than one source operator, the average value of outBfsize is calculated.

- Lines 5-12: Calculates the outBfsize value of all $B q_{i}$ operators to determine the corresponding partition of each operator. The algorithm computes the distance of the outBfsize of the operator with both average outBfsize of the source operators and outBfsize of the end operator. The partition $B q_{i}^{p 1}$ includes the operators of $B q_{i}$ which has the minimum distance with the source operators. Whereas, the partition $B q_{i}^{p 2}$ encompasses the $B q_{i}$ operators which has the minimum distance with the end operator.
Algorithm 1: $B q_{i}$ partitioning

Input: $B q_{i}$, query

Output: $B q_{i}^{\prime}$, partitioned query

1. sourceoperators $\leftarrow B q_{i}$. sources

2. endoperator $\leftarrow B q_{i}$. end

3. $s d f \leftarrow \operatorname{Avg}\left(\right.$ OutBfsize (sourceoperators ${ }^{\text {Inp }}$ ))

4. $d d f \leftarrow$ OutBfsize (endoperator ${ }^{\text {Out }}$ )

5. for (operator: TopologicalOrder $\left(B q_{i}\right)$ )

6. sdistance $\leftarrow \mid$ OutBfsize (operator $\left.^{I n p}\right)-s d f \mid$

7. ddistance $\leftarrow \mid$ OutBfsize (operator $\left.{ }^{I n p}\right)-d d f \mid$

8. if (sdistance $\leq$ ddistance)

9. $B q_{i}^{\prime}{ }^{\text {. }}$ operator ${ }^{p}={ }^{\prime} p 1^{\prime}$

10. if (sdistance $>$ ddistance)

11. $B q_{i}^{\prime}{ }^{\text {. }}$ operator ${ }^{p}={ }^{\prime} p 2^{\prime}$

12. end for

13. return $B q_{i}^{\prime}$

Algorithm 1. Bpartitioner, $B q_{i}$ Query partitioning algorithm

\subsubsection{Partial critical path algorithm}

A partial critical path (PCP) of query instance $B q_{i j}$ is a longest execution path ended to $B q_{i j}$ end operator and started from the one of the $B q_{i j}$ operators where the deadline of $B q_{i j}$ is not missed [33]. BCframework efficiently provisions and schedules each PCP operators to increase the likelihood of $B q_{i j}$ deadline meeting. Each PCP is calculated based on earliest start time (est), latest finish time (lft) and the minimal runtime of each $B q_{i j}$ operator instance demonstrated in $B q_{i j}$ runtime matrix.

The est $\left(o_{i t}\right)$ of an operator $o_{i t}$, represents the earliest time that operator $o_{i t}$ can start its execution and it occurs when all parents of such operator finish their executions as early as possible. The $l f t$ of an operator $o_{i t}, l f t\left(o_{i t}\right)$, indicates the latest time an operator $o_{i t}$ can finish its execution without violating the deadline of $B q_{i j}$ and it happens when all children of $o_{i t}$ are executed as late as possible. The est $\left(o_{i t}\right)$ and $l f t\left(o_{i t}\right)$ are formally defined by Eqs. (5) and (6) respectively, 
Algorithm 2: Partial critical path determination

Input: $B q_{i j}^{\prime}$, partitioned query instance

Output: $B q_{i j}^{\prime}$. $p c p s$, partial critical paths of the

partitioned query instance

1. $p c p \leftarrow \emptyset ;$ parent $\leftarrow-1 ; B p \leftarrow \emptyset$

2. toassign $\leftarrow B q_{i j}$. end

3. check $\leftarrow B q_{i j}$. end

4. parents $\leftarrow$ Unassignedparents $\left(B q_{i j}\right.$. end $)$

5. while (Top(toassign)! $=B q_{i j}$. end)

6. while (parents $!=$ null)

7. for ( $p$ : parents)

8. if $\left(p^{\text {est }}+p^{\text {runtime }}{ }^{\text {min }}+E_{\text {pcheck }}^{\text {Trf }}>\right.$ parent $)$

9. $\quad$ parent $\leftarrow p^{\mathrm{est}}+p^{\text {runtime }}{ }^{\mathrm{min}}+E_{\text {pcheck }}^{\mathrm{Trf}}$

10. $B p \leftarrow p$

11. end if

12. end for

13. $p c p \leftarrow B p \cup p c p$

14. toassign $\leftarrow$ parents $-B p$

15. check $\leftarrow$ Top $(p c p)$

16. parents $\leftarrow$ Unassignedparents (check)

17. end while

18. $B q_{i j} \cdot p c p s+=p c p$

19. $p c p \leftarrow \emptyset$

20. check $\leftarrow$ Top(toassign)

21. Remove Top(toassign) from the toassign

22. $p c p \leftarrow$ check

23. parents $\leftarrow$ Unassignedparents $($ check)

\section{4. end while}

25. return $B q_{i j}$. pcps

Algorithm 2. Partial Critical Path algorithm

$\operatorname{est}\left(O_{i t}\right)=\left\{\begin{array}{l}0, \quad f O_{i t}=O_{\text {source }} \\ \max _{t_{a} \in \operatorname{parents}\left(O_{i t}\right)}\left(\operatorname{est}\left(t_{a}\right)+\operatorname{Min}\left(R_{t_{a} k}^{j}\right)+E_{t_{a} O_{i t}}^{T r f}\right), \quad \text { Otherwise }\end{array}\right.$

$\operatorname{lft}\left(O_{i t}\right)=\left\{\begin{array}{l}B q_{i j}^{D L}, \quad \text { IfO } O_{i t}=\mathrm{O}_{\text {end }} \\ \max _{t_{s} \in \text { childs }\left(O_{i t}\right)}\left(\operatorname{lft}\left(t_{s}\right)-\operatorname{Min}\left(R_{t_{s} k}^{j}\right)-E_{O_{i t t_{s}}}^{T r f}\right), \quad \text { Otherwise }\end{array}\right.$

As shown in Algorithm 2, the PCP initially includes one of the parents of $O_{\text {end }}$ or, if it was already assigned, the operator with latest $l f t$ that has not been yet assigned to a PCP. The next element of PCP is the parent of the included operator with latest finish time which is calculated based on the earliest start time of the parent operator $p^{\text {est }}$, the minimum runtime of the parent operator $p^{\text {runtime }}{ }^{\text {min }}$ and the network traffic delay between the parent operator $p$ and candidate parent operator check, $E_{\text {pcheck }}^{\text {Trf }}$. The process is iterated for each parent of $O_{\text {end }}$.

\subsubsection{Provisioning and scheduling for original operator}

Oscheduler algorithm aims to decrease the number of provisioned public Cloud virtual machines and assign them to the original operator instances of the accepted query instance $B q_{i j}$ to decrease the average tuple latency, the public Cloud resource utilization cost and the deadline misses of $B q_{i j}$.

Algorithm3: Oscheduler: $\mathrm{Bq}_{i}$ original operator instances scheduling

Input: $B q_{i j}^{\prime} . p c p s$, partial critical paths of the

partitioned query instance

Output: Schedule, Schedule

1. $p 1 \leftarrow 0, p 2 \leftarrow 0 / /$ for counting the virtual slots of the first and second partitions respectively.

2. for ( $\left.p c p: B q_{i j} \cdot p c p s\right)$

3. if $\left(p c p^{\mathrm{p}}=p 1\right)$

4. $\quad$ vslot $=$ Vmtype (Fastest)

5. else

6. $\quad$ vslot $=$ Vmtype (Cheapest)

7. end if

8. $v_{\text {slot }}{ }^{\mathrm{st}}=\operatorname{Start}($ vslot $)$

9. $p c p^{\mathrm{st}}=\operatorname{Max}\left(p c p^{\mathrm{est}}, v_{\text {slot }}{ }^{\mathrm{st}}\right)$

10. schedule [Vm (vslot), pcp.operators $] \leftarrow 1$

11. $p c p^{\text {lag }}=\operatorname{Lag}(p c p)$

12. $p c p^{\text {Runtime }}=\operatorname{Runtime}(p c p)$

13. if $\left(\mathrm{pcp}^{\mathrm{p}}=p 1\right)$

14. vslot $_{\mathrm{p} 1++}^{\mathrm{p} 1}=$ vslot

15. Determine freeslots and add them to fvslo

16. else

17. vslot $_{\mathrm{p} 2++}^{\mathrm{p} 2}=$ vslot

18. Determine freeslots and add them to fvslo

19. end if

20. end for

Algorithm 3. Oscheduler algorithm

To provision public Cloud $V M s$, the algorithm applies two provisioning strategies for the original operator instances of $B q_{i j}$ identified partitions. For the first partition, the algorithm provisions the fastest $V M$ type of the underling public Cloud based on the highest $\mathrm{Vm}_{k}^{\mathrm{Cu}}$ and $V m_{k}^{R m}$ to improve the processing speed of the first partition operators. This strategy reduces the average tuple latency of these operators and provides the input $B f$ of their succeeding operators faster. For the second partition, the algorithm provisions the cheapest $V M$ type of the underling 
public Cloud based on the lowest $V m_{k}^{C t}$ to reduce the cost of the Cloud $V M$ instances utilization (Lines 1-7).

To assign $B q_{i j}$ original operator instances, the algorithm assigns all operators of each PCP in each partition to the corresponding provisioned $V m$ instance. This strategy can reduce the inter-operator traffic load of the operators and lead to reduce the average tuple latency of $B q_{i j}$. Furthermore, the algorithm adjusts the start time of the first operator of each assigned PCP to the maximum between the assigned operator est and assigned slot starttime. This adjustment can decrease the likelihood of $B q_{i j}$ deadline violations (Lines 8-10).

In addition, the algorithm determines the initial unassigned virtual machine slots, free $V m$ slots, which will be used by Rscheduler to replicate the operator instances. Therefore, the algorithm considers the runtime of the assigned PCPs to calculate the available free $V m$ slots. Furthermore, the free $V m$ slots are partitioned into two partitions by their VM types. More precisely, the free $\mathrm{Vm}$ slots of the first partition include unassigned fastest virtual slots and the second partition free $V m$ slots encompass unassigned cheapest virtual slots (Lines 13-19).

\subsubsection{Provisioning and scheduling for replicated operator}

Rscheduler algorithm aims to mitigate the performance variation of public Cloud virtual machines through the efficient replication of $B q_{i j}$ operator instances. To approach the goal, the algorithm efficiently determines the candidate $B q_{i j}$ operators which can be replicated, $O_{t}^{\text {tpe }=\text { replicated }}$, and assigned to the efficient candidate free $V m$ slots.

To efficiently determine the candidate operators for replication, the algorithm takes into account the structure and lag time of the $B q_{i j}$ operators to determine the operators whose position in the query are early and their lag time is minimum. As delay in the early operators execution causes delays of the bigger number of succeeding operators, prioritizing the early operators for replication may reduce the average tuple latency of $B q_{i j}$. Furthermore, prioritizing the minimal lag time operators can decrease the likelihood of $B q_{i j}$ deadline misses.

The candidate free $V m$ slots are determined by the following conditions (Lines 4-13).

- The candidate slot does not violate the earliest start time of PCP.

- PCP can complete its execution before the end of the candidate slot.

- The candidate slot is available after the precedent operators of PCP and before the succeeding operators of PCP.
- The $V m$ of the candidate slot does not host the corresponding original operators of PCP.

Algorithm 4: Rscheduler: $\mathrm{Bq}_{i}$ replicated operator instances scheduling

Input: $\mathrm{Bq}_{i j}$, query instance

Output: Schedule', Schedule

1. $t p c p \leftarrow \varnothing$

2. $B q_{i j} \cdot p c p s=\operatorname{Sort}\left(B q_{i j} \cdot p c p s\right.$, Lag, Asc $)$

3. $f v s l o t=\operatorname{Sort}(f v s l o t$, size, Desc $)$

4. for $\left(p c p: B q_{i j}\right.$. $\left.p c p s\right)$

5. if $\left(\operatorname{Min}\left(\mathrm{U}_{i 1=1}^{\left|B q_{i j} \cdot p c p s\right|} B q_{i j} \cdot p c p_{i 1}^{\text {runtime }}\right)>\right.$ $\left.\operatorname{Max}\left(\sum_{k 1=1}^{|f v s l o t|} f_{v s l o t}\right)\right)$ break

6. for (slot: fvslot)

7. if $\left(p c p^{\mathrm{p}=\mathrm{p} 1}\right.$ and $\left.s l o t^{\mathrm{p} \neq \mathrm{p} 2}\right)$

8. if $\left(s l o t^{\text {st }}+p c p^{\text {runtime }}<p c p^{\text {lft }}\right)$

9. if $(V M($ slot $)$.operators $\cap$ pcp.operators $==$ null)

10. if $\left(\operatorname{slot}^{\mathrm{st}} \geq(\operatorname{prec}(p c p))^{\mathrm{ft}}\right.$ and $\operatorname{slot} \mathrm{ft}^{\mathrm{ft}} \leq$ $\left.(\operatorname{succ}(p c p))^{\mathrm{st}}\right)$

11. if $\left(s l o t^{\text {time }} \geq p c p^{\text {runtime }}\right.$ and $p c p^{\text {est }} \geq$ slot $\left.^{\text {st }}\right)$ )

12. candidateslots $+=$ slot

13. end for

14. if (candidateslots $==$ null and $p c p$ ! = null)

15. $\mathrm{Bq}_{i j} \cdot p c p s-=p c p$

16. $p c p-=\operatorname{Last}(p c p$.operators)

17. $\mathrm{Bq}_{i j} \cdot p c p s+=p c p$

18. $t p c p+=$ Last(pcp.operators)

19. $\mathrm{Bq}_{i j}$. $p c p s+=t p c p$

20. else if (candidateslots! = null )

21. $B s l o t=\operatorname{Min}\left(\cup_{k 2=1}^{j} \mid\right.$ candidateslots ${ }_{k 2}^{\text {start }}-$ pcp ${ }^{\text {start }} \mid$ )

22. schedule $^{\prime}[$ Vm (Bslot), pcp.operators $] \leftarrow 1$

23. $B$ slot $-=p c p^{\text {runtime }}$

24. $\mathrm{Bq}_{i j} \cdot$ pcps ${ }^{\prime}+=p c p$

25. if $(B s l o t \neq$ null $)$

26. Add Bslot into fvslot

27. else

28. Remove Bslot from fvslot

29. end if

30. end if

31. end for

Algorithm 4. Rscheduler algorithm

The algorithm efficiently selects the candidate free $\mathrm{Vm}$ slots based on the strategy which reserves the fastest candidate slots for the replicated operators of first partition and reserves the largest candidate slots for the replicated 
operators of both partitions. Using this strategy can improve the processing speed of the operators of PCP in the first partition and provide the largest remained free $\mathrm{Vm}$ slots, not assigned slots with maximum free slots, which may be assigned to the most operators of an unassigned PCP.

The efficient assignment of the determined free $\mathrm{Vm}$ slots to the candidate operators is achieved through obeying a defined rule which state that the PCP whose operators are the member of the first partition of $B q_{i j}$, first partition PCP, must be assigned to $V m$ slots which are fastest. For a second partition PCP, it can be assigned to both first or second free $V m$ slots. In a situation where it is possible to assign the first partition free $V m$ slots to both first and second partition PCPs, the priority of the first partition PCP is higher (Lines 20-24). This rule aims to minimize the delay of the PCP start time.

The assignment may create new smaller free slots when the assigned PCP size is smaller than the assigned free slot. The algorithm adds these new slots to the list of the available free slots (Lines 24-29). Furthermore, for a PCP the last instance of $B q_{i}$ whose deadline violation is maximum to determine its $V m$ type. The last instance of $B q_{i}$ and maximum deadline violations are determined by First function and descending (Desc) parameter in Sort function respectively. This strategy can lead to provide the new free $V m$ slots which will be used by Rscheduler to schedule the PCPs like the identified PCP to the new added virtual slots.

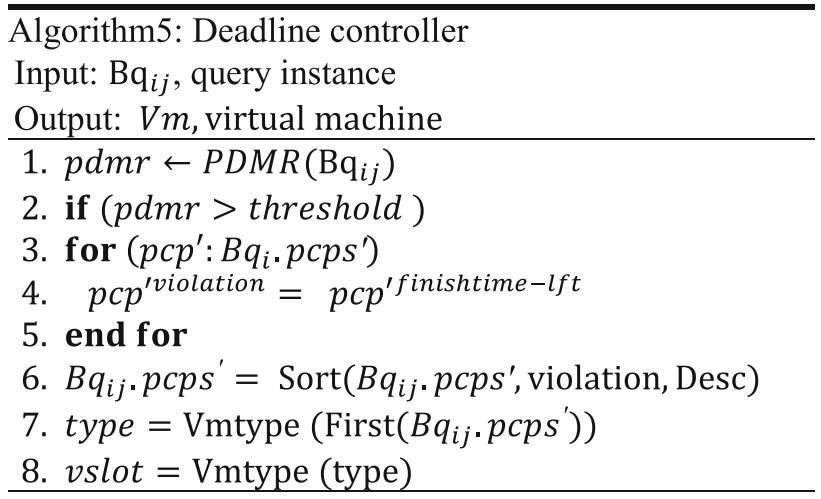

$\operatorname{PDMR}\left(B q_{i j}\right)=\frac{\sum_{j 1=1}^{j 1=\mid \text { missedinstnces } \mid}\left(B q_{i j 1}^{w}-\sum_{q=1}^{q=\text { windowsize }} \sum_{r=1}^{r=\left|D s_{p}\right|} ! \text { deadlinemiss }\left(D s_{q r}\right)\right) / B q_{i}^{w}}{\sum_{j=1}^{j=\mid \text { executedinstnces } \mid} B q_{i j}}$

which there is no free $V m$ slots, the algorithm removes at least operators of the PCP to find a free slot to fit the modified PCP (Lines 14-19). In addition, the algorithm inserts the new created PCP into the list of accepted PCPs.

\subsubsection{Deadline controller}

The aim of the deadline controller algorithm is to reduce the likelihood of missing the $B q_{i j}$ deadline, $B q_{i j}^{D L}$, through incrementally provisioning $V m$ instances required for $B q_{i j}$. To reach the goal, the algorithm evaluates the missed deadline ratio of the previously executed query instances to provision an appropriate virtual machine for the next execution instance of $B q_{i j}$.

The algorithm calculates the ratio of the proportional deadline miss using the Eq. (7) [2] which prioritizes deadline missed query instances whose missed deadline tuples of their window are high.

Using the $P D M R$ value of $B q_{i}$, the algorithm provisions the appropriate new type of $V m$ when the $P D M R$ value is greater than the predefined threshold. To determine the appropriate $V m$ type, the algorithm examines the PCP of
Figure 3 shows the simplified schedule Schedule' for instance $j$ of $B q_{i j}$ where its deadline is twenty. As shown in Fig. 3a, there are four PCPs with a simplified estimated runtime which are identified based on the output $B f$ size of the operators and partitioned into two partitions P1 and P2. As shown in Fig. 3b, two $V m$ types $V m F$, fastest $V m$, and $\mathrm{VmC}$, cheapest $V m$, and four $V m$ instances are provisioned

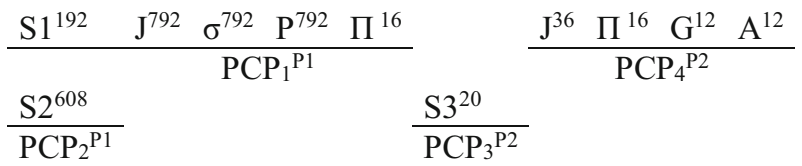

(a)

\begin{tabular}{|c|c|c|c|c|c|}
\hline$V m_{1}{ }^{\mathrm{F}}$ & $\mathrm{PCP}_{1}^{\mathrm{P} 1}(10)$ & $\mathrm{PCP}_{3}{ }^{, P 2}$ & & & \\
\hline$V m_{2}{ }^{\mathrm{F}}$ & $\mathrm{PCP}_{2}{ }^{\mathrm{Pl}}(5)$ & $\mathrm{PCP}_{1}{ }^{, \mathrm{P} 1}$ & & $\mathrm{PCP}_{4}{ }^{, \mathrm{P} 2}$ & \\
\hline$V m_{1}{ }^{\mathrm{C}}$ & & $\mathrm{PCP}_{3}{ }^{\mathrm{P} 2}(2)$ & & & \\
\hline$V m_{2}{ }^{\mathrm{C}}$ & & & $\mathrm{PCP}_{4}$ & $2(4)$ & \\
\hline Time & 10 & 12 & 15 & $\begin{array}{lll}16 & 19\end{array}$ & 20 \\
\hline
\end{tabular}

(b)

Fig. 3 Scheduling and provisioning of $B q_{i j}$. a Instance $j$ of $B q_{i}^{\prime}$ and its PCPs. b Schedule' of $B q_{i j}$ 
and their possible free $\mathrm{Vm}$ slots are used to replicate the operators of three PCPs including PCP1'P1, PCP3'P2,PCP4'P2.

\subsection{Complexity analysis}

The main cost of the partitioning algorithm comes from the sorting and traversing $B q_{i}$ operators. Therefore the time complexity of the algorithm is $O($ on $\log o n+o n)$, where on represents the number of $B q_{i}$ operators. In partial critical path algorithm, the computation load is primarily made up of traversing $B q_{i j}$ operators. As a result the time complexity of the algorithm for each query instance is $O(o n)$.

For Oscheduler, the main step is to provision the $V m$ $\mathrm{s}$ for all available PCPs. So, the time complexity of the examining PCPS is $O(|P C P s|)$. Rscheduler sorts both PCPs and free $V m$ slots and examines all virtual slots to determine the possible and efficient free $V m$ slots for a PCP. Therefore, the time complexity of this algorithm is $O(|\mathrm{PCPs}| \log |\mathrm{PCPs}|+\mid$ fvslot $|\log |$ fvslot $\mid+$ $\mid$ PCPs $|\times|$ fvslot $\mid)$. In addition, as the deadline controller algorithm evaluates and sorts the replicated PCPs, the time complexity of this algorithm is $O(\mid \mathrm{PCP}$ 's $\mid$ $\log \mid$ PCP's $|+|$ PCP's $\mid$ ).

\section{Evaluation}

This section describes the experiments that have been performed to validate and analyze the performance of BCframework. First, the query partitioning algorithm of BCframework is validated by evaluating outBfsize of identified partitions during execution of the benchmark and real-world queries. Then, BCframework performance is assessed by analyzing the average tuple latency, the utilization cost, the number of deadline misses and the number of provisioned $\mathrm{Vm}$ instances for the benchmark and real-world queries in presence of different $B s$ fluctuation scenarios.

The performance of BCframework is compared with Storm default scheduler under simple and complex queries because it is one of the most popular Big Data stream computing platforms both in academia and industry [30]. Furthermore, the performance of the BCframework is also compared under real-world queries with a state-of-the-art scheduler [30] because of its similarity. Similar to the problem statement of BCframework, the state-of-the-art scheduler addresses the problem of provisioning and scheduling Big Data stream applications and its framework is based on a cost and deadline aware scheduler [34].

BCframework is evaluated on the simulated Storm platform [7] and Amazon EC2 [35] public Cloud while not limited to these infrastructures. As the data and query models of BCframework are developed based on Direct Acyclic Graph (DAG), BCframework is compatible to other DAG-based distributed stream processing platforms like Apache Spark Streaming [36] and schedule model of BCframework can be deployed on these systems. $\mathrm{BCframework}$ is also portable to other cloud providers as there is no assumption about the specification of a particular Cloud provider except that the Cloud provider pricing model should be imported to BCframework.

\subsection{Queries}

The benchmark queries used in the experiments have been applied from the BigBench [37] and Linear Road Benchmark [38] benchmarks. In addition, two real-world queries WordCount [39] and Top_N [40] are also used in the experiments.

BigBench includes the complex queries which cover the declarative and procedural and also the structured, semistructured and unstructured aspects of the Big Data analytic queries. The characteristics of the BigBench queries are described in Table 2.

The volume of BigBench queries is supported by a parallel data generator which is able to generate data in scale, the velocity of BigBench queries is covered with data clicks and sensor data at an increasing rate and the variety of BigBench is supported by the structural relational tables, semi-structured key-value web-clicks and unstructured social data text. The workload of BigBench is covered with 30 queries which covers five major area of Big Data analytics. Each structured, semi-structured and unstructured data types and their combination are covered in the queries [42].

As BigBench queries include SQL-like and the userdefined operators and also, the structured, semi-structured and the unstructured data types, it is chosen to evaluate BCframework as a scheduler for Big Data scenarios. The

Table 2 The characteristics of BigBench queries [37]

\begin{tabular}{llll}
\hline Query type & Queries & Data types & Queries \\
\hline Declarative & $6,7,9,13,14,16,17,19,21,22,23,24$ & Structured & $1,6,7,9,13,14,15,16,17,19,20,21,22,23,24,25,26,29$ \\
Mixed & $1,4,5,8,11,12,15,18,20,25,26,29,30$ & Semi-structured & $2,3,4,5,8,12,30$ \\
Procedural & $2,3,10,27,28$ & Unstructured & $10,11,18,27,28$ \\
\hline
\end{tabular}


fluctuating scenarios of our experiments are conducted by controlling the data generation rate of BigBench. Furthermore, as BigBench is targeted Cloud online services [46] we used it to evaluate the diversity of analytical queries in public Cloud.

Linear Road Benchmark queries encompass seventeen simple stream queries whose operators are SQL-like and their data types are structured. As Linear Road Benchmark has been used as benchmark for the evaluation of the distributed stream management systems, it is chosen to evaluate BCframework as a scheduling framework on distributed stream processing systems.

Real-world query, WordCount counts the number of words in a sentence and TOP_N, other real-world query, does a continuous computation of the top $\mathrm{N}$ words that the query has seen in terms of cardinality. These queries are used to evaluate BCframework performance in comparison with the state-of-the-art scheduler.

\subsection{Environment}

Table 3 describes the simulation test bed used in the experiments to run the queries. The test bed consists of a data center containing 20 hosts. Each host has $256 \mathrm{~GB}$ of RAM and 8 cores. Based on the pricing model of Amazon AWS EC2, there is no a utilization cost for the inter virtual machine network traffic but the utilization cost of the incoming and outgoing traffic loads for the zone of the leased virtual machines is calculated.

The our simulation conducted using CEPSim [43] which is an extension to CloudSim [44] and is used for the simulation of Complex Event Processing and Stream processing systems on different deployment models including private, public, hybrid and multi-clouds. It can be used to analyze the performance and scalability of user-defined queries and to evaluate the effects of various query processing strategies. CEPSim can simulate the systems in large Big Data scenarios with accuracy and precision [41].

To implement the queries, a software stack is used which includes Windows 7 64-bit, Jdk 1.8 and CEPSim simulator [45] and is executed on an Intel Dual-Core CPU

Table 3 Test bed specifications

\begin{tabular}{lllll}
\hline VM type & Core speed (ECU) & Memory (GB) & Cores & Cost $(\$)$ \\
\hline m1.small & 1 & 1.7 & 1 & 0.06 \\
m1.medium & 2 & 3.75 & 1 & 0.12 \\
m1.large & 2 & 7.5 & 2 & 0.24 \\
m1.xlarge & 2 & 15 & 4 & 0.48 \\
m3.xlarge & 3.25 & 15 & 4 & 0.50 \\
m3.xxlarge & 3.25 & 30 & 8 & 1.00 \\
\hline
\end{tabular}

3.0 GHz 64-bit with 4 GB RAM. Implemented queries are also available in the repository. ${ }^{1}$

\subsection{Set-up}

Table 4 describes the parameters used by BCframework to execute the queries.

Performance degradation model of public Cloud presented by [6] is used to conduct the experiments. Loss in performance observed in $V m$ for a specific scheduling period is sampled from a normal distribution with the average of $15 \%$ loss and standard deviation of $10 \%$ loss. Similarly, loss in each data transfer is modeled with uniform distribution with average of $30 \%$ loss and standard deviation of $10 \%$ loss.

For each query instance $B q_{i j}$ and each level of $B s^{V e}$, a soft deadline was generated as follows (adapted from [33]). A base runtime is defined as the execution time obtained with a provisioning and scheduling strategy which assigns each operator of the query to an instance of the most powerful $\mathrm{Vm}$ instance and assumes there is no inter node traffic cost. The base value obtained with such strategy is then multiplied by the number of operators. In addition, a deadline factor $\alpha$ is defined which is set by our conducted experiments. Therefore, the deadline of query $B q_{i j}$ is set by a.base.

The same random number generation seeds were used for each strategy, which ensures that the condition faced by each algorithm is exactly same regarding infrastructure behavior. Furthermore, the average of output metrics is presented in the following experimental result tables and figures.

The range of values for $B s^{V e}$, is set to evaluate BCframework in face of the fixed low rate and high fluctuating rate of the incoming Big Data streams. Furthermore, the value of $d s$ is also set to reflect the behavior of the windows-based operators of the benchmark and realworld queries. The range of values for $B s^{V a}$ is set using the structure of benchmark queries to evaluate BCframework algorithms in face of the different tuple sizes. The value of Threshold and $\alpha$ parameters are determined by the conducted experiments. Each query is run for $15 \mathrm{~min}$ so Execution and Period parameters are set to $15 \mathrm{~min}$.

\subsection{BCframework validation}

In order to validate BCframework, we validate the functionality of the query partitioning algorithm under the simple, complex benchmark and real-world queries. The validation is conducted by evaluating the identified partitions during their executions. In order to compare the

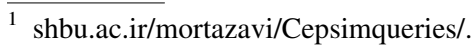


Table 4 Parameters used in BCframework

\begin{tabular}{lll}
\hline Parameter & Definition & Value \\
\hline$B s^{V e}$ & $B f$ fixed average rate $B f$ fluctuating average rate level & $10^{3}$ tuples/second $\left[10^{4}, 15 \times 10^{3}, 30 \times 10^{3}\right]$ tuples/second \\
$d s$ & Number of data sets based on window size $\left(B q_{i}^{W}\right)$ & $30 \mathrm{~s}$ \\
$B s^{V a}$ & Tuple size & {$[4 \mathrm{~B}-10 \mathrm{~KB}]$} \\
Execution & Estimated execution period & $15 \mathrm{~min}$ \\
Period & Billing period of $V M$ & $15 \mathrm{~min}$ \\
$\alpha$ & Deadline factor of query & {$[2-6]$} \\
Threshold & Deadline miss threshold & $50 \%$ \\
\hline
\end{tabular}

Table 5 Partitioning of Linear Road Benchmark queries

\begin{tabular}{llllllllllll}
\hline $\mathrm{Q}$ & $\begin{array}{l}\mathrm{R} \\
(\%)\end{array}$ & $\mathrm{P}$ & $\mathrm{Q}$ & $\begin{array}{l}\mathrm{R} \\
(\%)\end{array}$ & $\mathrm{P}$ & $\mathrm{Q}$ & $\begin{array}{l}\mathrm{R} \\
(\%)\end{array}$ & $\mathrm{P}$ & $\mathrm{Q}$ & $\begin{array}{l}\mathrm{R} \\
(\%)\end{array}$ & $\mathrm{P}$ \\
\hline 1 & 1.3 & 2 & 6 & 49.8 & 2 & 11 & 31.1 & 2 & 16 & 10.1 & 2 \\
2 & 51.7 & 2 & 7 & 27.1 & 2 & 12 & 44.2 & 2 & 17 & 17.7 & 2 \\
3 & 56.1 & 2 & 8 & 26.2 & 2 & 13 & 57.8 & 2 & & & \\
4 & 0 & 1 & 9 & 49.9 & 2 & 14 & 18.1 & 2 & & & \\
5 & 4.8 & 2 & 10 & 39.2 & 2 & 15 & 26.9 & 2 & & & \\
\hline
\end{tabular}

identified partitions, the ratio between the average outBfsize of the first partition and the average outBfsize of the second one is calculated and shown in the R column of the result tables. Furthermore, the number of identified partitions is reported in the $\mathrm{P}$ column of the following tables.

\subsubsection{Simple queries}

Table 5 shows the result of partitioning Linear Road Benchmark queries during execution of the queries. To execute these experiments, the configuration parameters $B s^{V e}$ and $B s^{V a}$ are set to [10,000-30,000 tuples/s] and [4B$45 \mathrm{~B}$ ] respectively, which later is derived from the structure of the Linear Road Benchmark queries.

The Table 5 demonstrates that the most Linear Road Benchmark queries are partitioned in two partitions but the average rate for these identified partitions is low. Since the distance of the tuple size of the first partition operators from the second one is small, the rate remains low. In addition, the table notes that, for the query Q4, the number of identified partitions is 1 which is affected by the simple structure of the query so all operators of Q4 are partitioned in the first partition.

\subsubsection{Complex queries}

Table 6 shows the result of partitioning BigBench queries during the execution of the queries. To execute these experiments, the configuration parameters $B s^{V e}$ and $B s^{V a}$ are set to $[10,000-30,000$ tuples/s] and [500B-10 KB]
Table 6 Partitioning of BigBench queries

\begin{tabular}{|c|c|c|c|c|c|c|c|c|}
\hline Q & $\begin{array}{l}\mathrm{R} \\
(\%)\end{array}$ & $\mathrm{P}$ & Q & $\begin{array}{l}\mathrm{R} \\
(\%)\end{array}$ & $\mathrm{P}$ & Q & $\begin{array}{l}\mathrm{R} \\
(\%)\end{array}$ & \\
\hline \multicolumn{9}{|c|}{ (a) Queries 1-15 } \\
\hline 1 & 97.1 & 2 & 6 & 85.4 & 2 & 11 & 97.4 & \\
\hline 2 & 61.1 & 2 & 7 & 98.1 & 2 & 12 & 83.1 & \\
\hline 3 & 94.3 & 2 & 8 & 68.1 & 2 & 13 & 61.9 & \\
\hline 4 & 45.7 & 2 & 9 & 97.1 & 2 & 14 & 97.2 & \\
\hline 5 & 91.1 & 2 & 10 & 0 & 1 & 15 & 94.1 & \\
\hline \multicolumn{8}{|c|}{ (b) Queries 16-30 } & \\
\hline 16 & 94.1 & 2 & 21 & 37.4 & 2 & 26 & 90.3 & \\
\hline 17 & 98.8 & 2 & 22 & 88.6 & 2 & 27 & 0 & \\
\hline 18 & 80.9 & 2 & 23 & 82.8 & 2 & 28 & 74.1 & 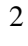 \\
\hline 19 & 91.3 & 2 & 24 & 92.1 & 2 & 29 & 95.9 & 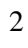 \\
\hline 20 & 84.1 & 2 & 25 & 90.2 & & 30 & 89.9 & 2 \\
\hline
\end{tabular}

respectively, which later is derived from the structure of the BigBench queries.

As shown in Table 6, for the most BigBench queries, they are partitioned into two partitions and their rates are high. The high rate of the identified partitions is due to this fact that the distance of the tuple size of the first partition operators from the second one is large. In particular, Q17 has the highest rate which is a caused by the complexity of its structure and the number of its operators. Meanwhile, for queries like Q4, the rate is low which is affected by its linear structure. In addition, for the queries Q10, Q27 the number of identified partitions is 1 which is affected by the simple structure of the query so all operators of each query are partitioned in the first partition.

\subsubsection{Real-world queries}

The result of the partitioning of the WordCount and TOP_N queries is plotted in Fig. 4. The needed configuration parameters $B s^{V e}$ and $B s^{V a}$ are set to $[10,000-30,000$ tuples/s] and [4B-800B] respectively, which later are derived from the structure of the queries. 


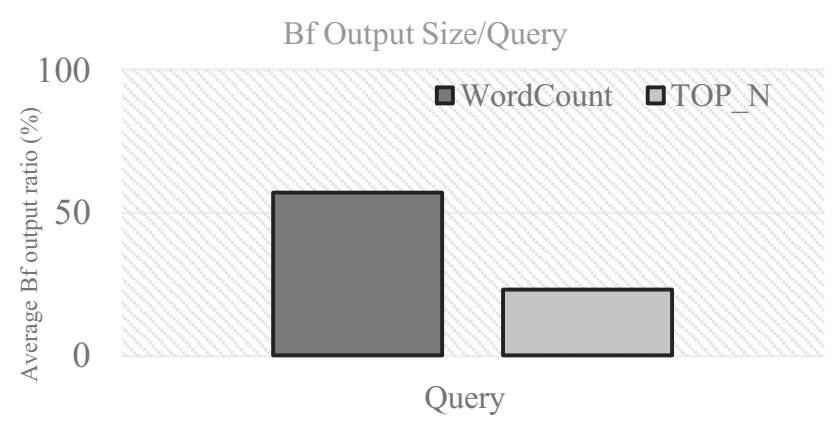

Fig. 4 Result of partitioning two real-world queries

As shown in Fig. 4, the average outBfsize ratio between the first partition and second one for the WordCount query is higher than the TOP_N query because the structure of WordCount includes more complex operators than Top_N query.

\subsection{BCframework performance}

In order to evaluate BCframework performance, we compare the result of the average tuple latency, the utilization cost, the number of the deadline misses and the number of provisioned $V m$ instances achieved by Storm default scheduler (the default scheduler) with BCframework under the simple and complex benchmark queries. In addition, BCframework performance is compared with the state-ofthe-art scheduler [30] under the real-world queries. All these experiments have been performed in the presence of fixed and fluctuating $B s$ scenarios. Therefore, the configuration parameter $B s^{V e}$ is set to 1000 tuples/s and [10,000-30,000 tuples/s] for fixed and fluctuating scenarios respectively.

The following tables and figures show the experimental results in presence of fixed and fluctuating rate in their $\mathrm{FX}$ and FL symbols respectively by using BCframework (BC) algorithms, Storm default scheduler (DF) or the state-ofthe-art scheduler (ST). The observed output metrics tuple latency, utilization costs, total number of deadline violations and the number of provisioned $V m$ instances are reported for each conducted experiment.

\subsubsection{Simple queries}

Figure 5 shows the average tuple latency of a sample Linear Road Benchmark query during the execution time of fifteen minutes. The average tuple latency reduction of all Linear Road Benchmark queries is presented in Fig. 6. The experiments are performed by the configuration parameter $B s^{V a}$ set to [4B-45B] based on the each Linear Road Benchmark query structure. In addition, the deadline factor $\alpha$ is set to $[2,3]$.

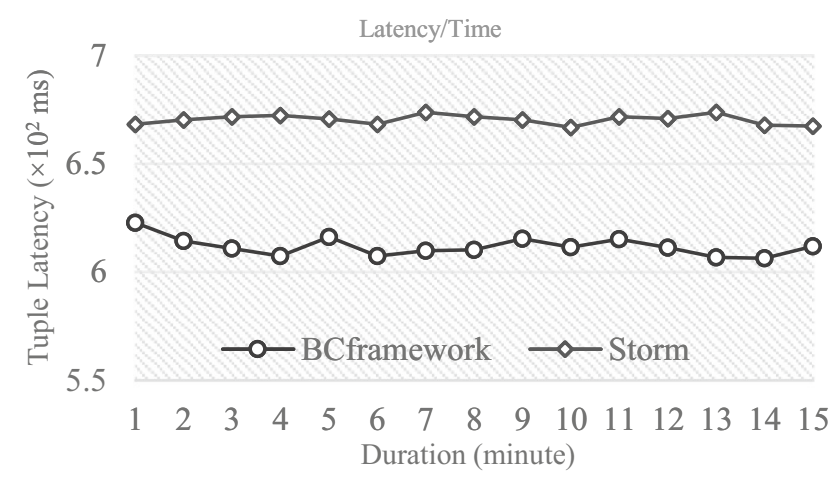

(a)

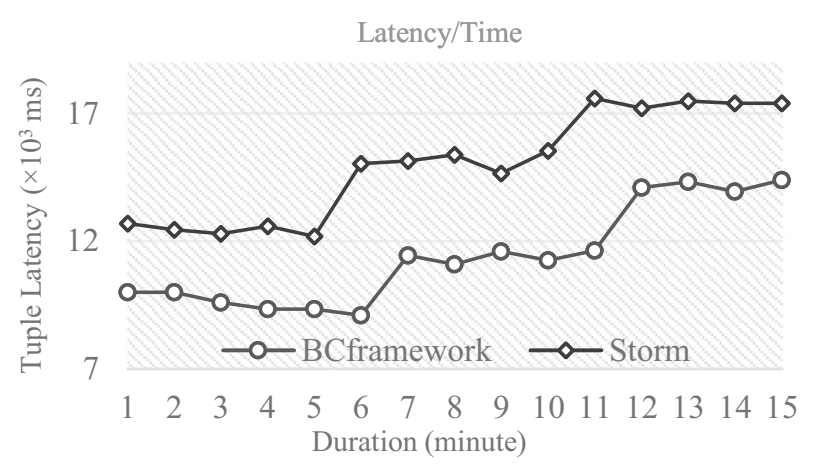

(b)

Fig. 5 Average tuple latency reduction of Linear Road Benchmark Query Q8. a Fixed rate. b Fluctuated rate

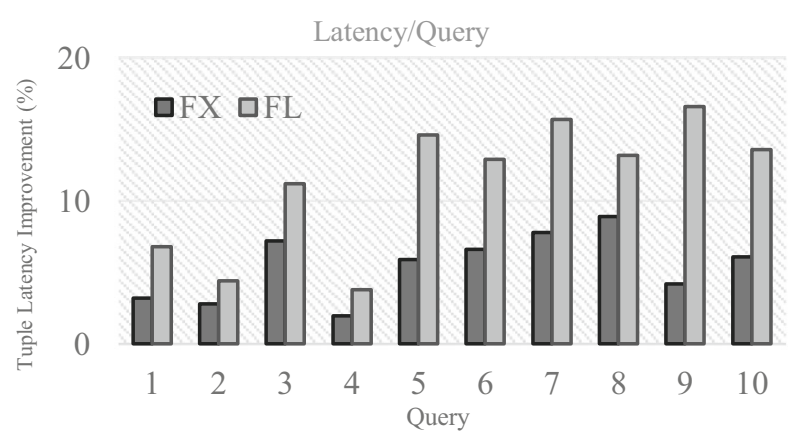

(a)

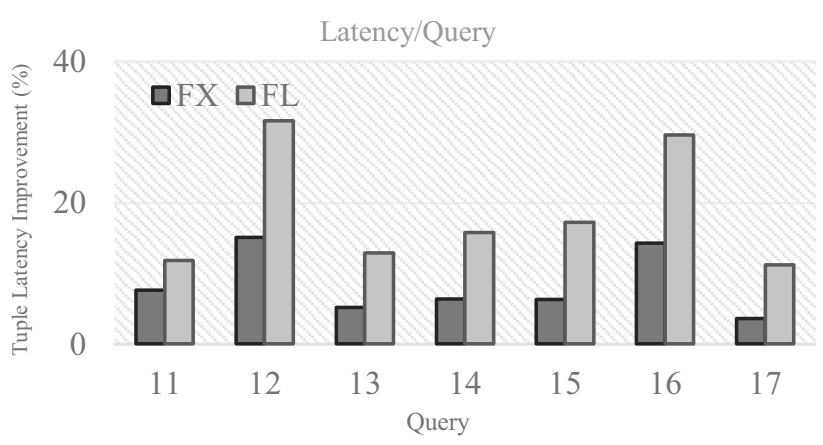

(b)

Fig. 6 Average tuple latency improvement under Linear Road Benchmark by BCframework. a Queries 1-10. b Queries 11-17 
As shown in Fig. 5a and b, for query Q8 an average tuple latency reduction of $8.9 \%$ and $13.2 \%$ is achieved respectively. The improvement in Q8 is related to its structure where two PCPs are identified by BCframework. These enable Oscheduler to provision one instance of the fastest VM type and one instance of the cheapest VM type to assign them to the operators of PCPs. Whereas, the default scheduler does not take into account Q8's structure and the type of VM and simply distributes Q8 operators.

Figure 6 shows that BCframework only yields latency improvement of about $4 \%$ for the most Linear Road Benchmark queries like Q2 and Q4. This can be caused by the linear structure of the queries, the small numbers of identified PCPs and the short length of identified PCPs.

Figure 7 shows the average cost reduction of the Linear Road Benchmark queries. For these queries, the length of PCPs in the second partition is short and as a result BCframework's VM utilization strategy can only be applied for a small number of operations in the second partition. Whereas, for a given set of the cheapest VM instances, the default scheduler is able to assign the VM instances to all operators of the queries.

Table 7 shows that the BCframework either completely eliminates deadline violations of the simple queries or reduces them significantly. The BCframework's deadline control mechanism monitors the deadline violation of

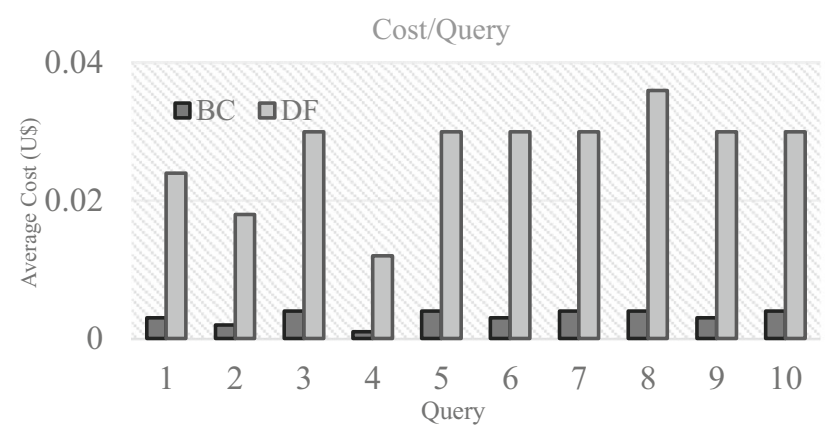

(a)

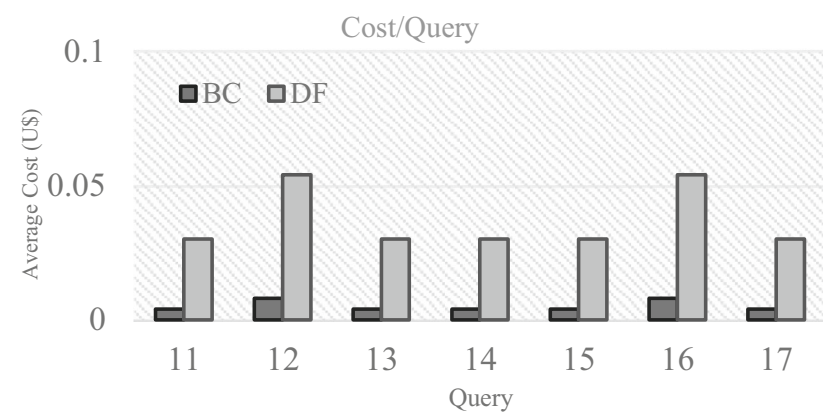

(b)

Fig. 7 The average cost (U\$) under Linear Road Benchmark a Queries 1-10. b Queries 11-17 simple queries continuously whereas the default scheduler does not take into account the deadline of the queries.

Figure 8 shows that BCframework outperforms the default scheduler in $\mathrm{Vm}$ instance provisioning under simple queries. The default scheduler assigns each operator of a query to a provisioned $V m$ instance while BCframework provisions $V m$ instances by considering structure of the queries and applies different provisioning strategies for a query. For query Q8, BCframework has identified two PCPs in the partitions and provisions two instances where the default scheduler uses six provisioned instances for six operators of Q8. For query Q16, BCframework and the default scheduler assign the same number of $\mathrm{Vm}$ instances because BCframework provisions five $\mathrm{Vm}$ instances for five identified PCPs and the default scheduler distributes nine operators of the query to all five provisioned instances.

\subsubsection{Complex queries}

Figure 9 shows the average tuple latency of the sample BigBench query during their 15 min execution. The average tuple latency reduction of all BigBench queries is presented in the Fig. 10. These experiments are performed

Table 7 Total number of deadline violations under Linear Road Benchmark queries

\begin{tabular}{|c|c|c|c|c|c|c|c|c|c|}
\hline$B s^{V e}$ & Q & $\alpha$ & $\mathrm{BC}$ & DF & $B s^{V e}$ & Q & $\alpha$ & $\mathrm{BC}$ & DF \\
\hline \multicolumn{10}{|c|}{ (a) Queries 1-10 } \\
\hline FX & 1 & 3 & 0 & 0 & FX & 6 & 3 & 0 & 0 \\
\hline FL & & 3 & 2 & 8 & FL & & 3 & 1 & 7 \\
\hline FX & 2 & 3 & 0 & 0 & FX & 7 & 3 & 0 & 0 \\
\hline FL & & 3 & 1 & 8 & FL & & 3 & 1 & 8 \\
\hline FX & 3 & 3 & 0 & 0 & FX & 8 & 3 & 0 & 2 \\
\hline FL & & 3 & 1 & 9 & FL & & 3 & 3 & 10 \\
\hline $\mathrm{FX}$ & 4 & 3 & 0 & 0 & FX & 9 & 3 & 0 & 0 \\
\hline FL & & 3 & 3 & 9 & FL & & 3 & 2 & 8 \\
\hline FX & 5 & 3 & 0 & 0 & $\mathrm{FX}$ & 10 & 3 & 0 & 0 \\
\hline FL & & 3 & 2 & 9 & FL & & 3 & 3 & 9 \\
\hline \multicolumn{10}{|c|}{ (b) Queries 11-17 } \\
\hline FX & 11 & 3 & 1 & 3 & FX & 16 & 2 & 2 & 6 \\
\hline FL & & 3 & 2 & 10 & FL & & 3 & 2 & 14 \\
\hline FX & 12 & 3 & 0 & 0 & FX & 17 & 2 & 0 & 0 \\
\hline FL & & 3 & 1 & 12 & FL & & 3 & 1 & 7 \\
\hline FX & 13 & 3 & 0 & 0 & & & & & \\
\hline FL & & 3 & 2 & 9 & & & & & \\
\hline FX & 14 & 3 & 0 & 0 & & & & & \\
\hline FL & & 3 & 2 & 8 & & & & & \\
\hline FX & 15 & 3 & 0 & 0 & & & & & \\
\hline FL & & 3 & 3 & 9 & & & & & \\
\hline
\end{tabular}




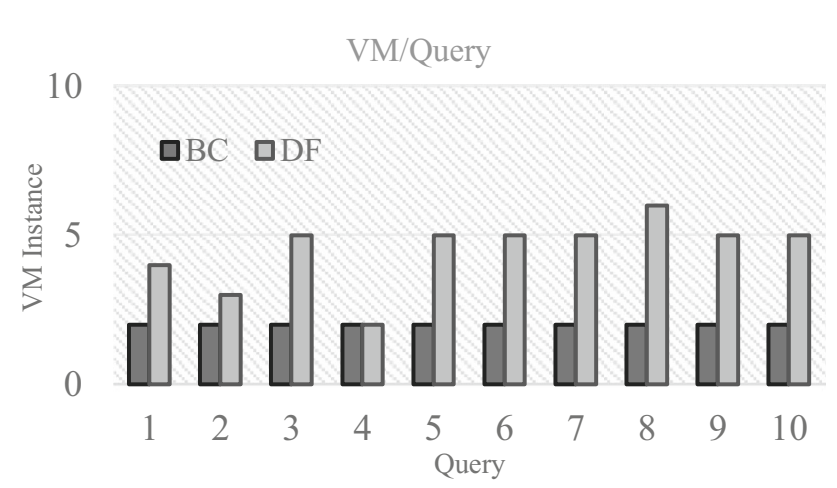

(a)

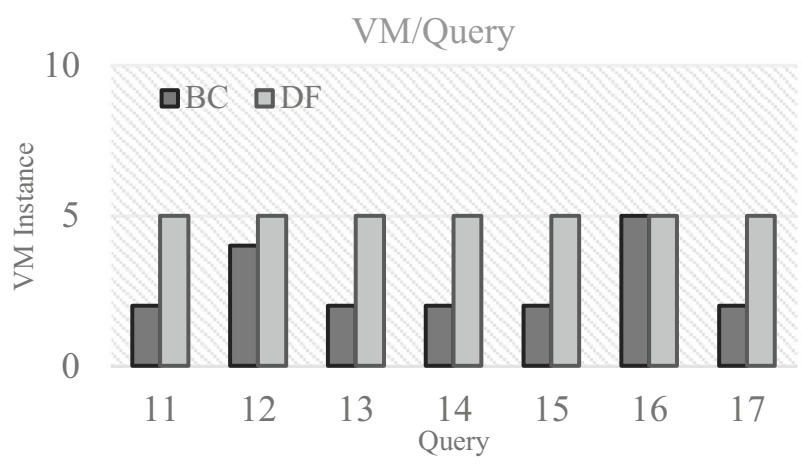

(b)

Fig. 8 The number of provisioned $V m$ instances under Linear Road Benchmark a Queries 1-10. b Queries 11-17

with the configuration parameter $B s^{V a}$ set to [500B-10 KB] based on the structure of BigBench queries.

Figure $9 \mathrm{a}$ and $\mathrm{b}$ show that $\mathrm{BCframework}$ yields latency improvement of $16.2 \%$ and $46.8 \%$ respectively for the query Q1. The improvement of Q1 is related to its complex structure where BCframework identified more than one PCP in each partition of the query Q1. Oscheduler assigns all original operators of the identified PCP to a $V m$ instance and Rscheduler assigns the provided free $\mathrm{Vm}$ slots to the Q1 selected replicated operators. Whereas, the default scheduler does not take into account the Q1 structure, VM types and does not provide any scheduling strategy for replicated operators.

As shown in Fig. 10, the achieved improvement for the BigBench queries varies between 7.2 and $65.8 \%$ which might be caused by three main reasons: First, the structure of these queries encompasses the different styles (i.e., simple linear style or more complex star and diamond patterns or even a mix of them).

For simple linear queries like Q2 the most BCframework optimizations are not applicable while BCframework optimizations can be completely applied to complex queries like Q13. Second, the number of query operators is significantly different. For some queries like Q19, there are more than 30 operators which lead to the better

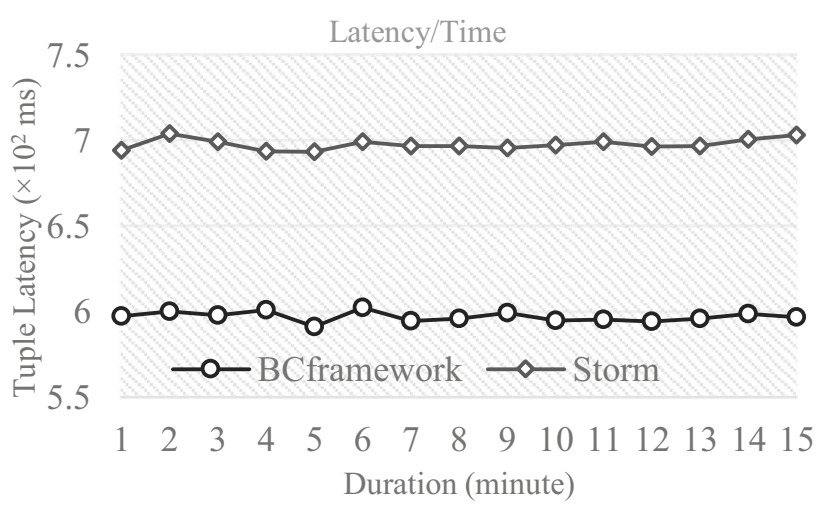

(a)

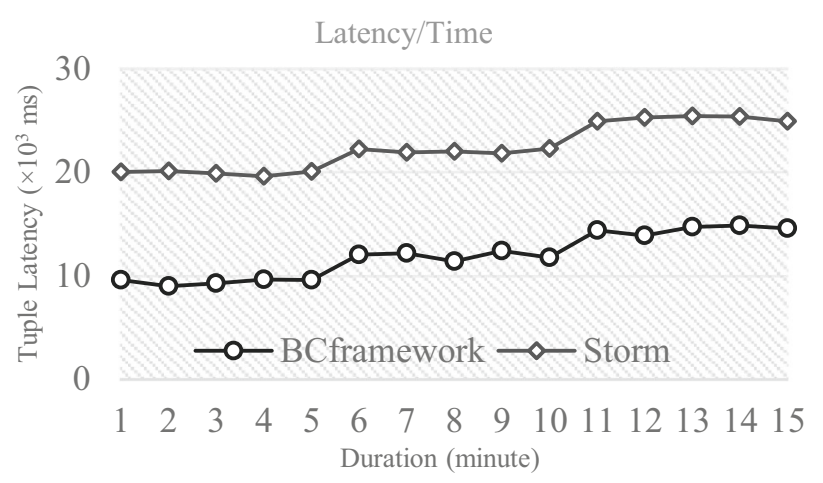

(b)

Fig. 9 Average tuple latency reduction of BigBench query Q1

performance improvement achieved by the framework. Third, the variance tuple size $B s^{V a}$ of these queries can lead to their variable tuple latency improvement. For example, the tuple size of Q6 is about $2 \mathrm{~KB}$ larger than that of Q5 which increases traffic load incurred by Q6. Therefore, traffic load reduction techniques of BCframework lead to better tuple latency reduction.

Figure 11 demonstrates that the average cost reduction of the most BigBench queries like Q2 and Q4 is about $90 \%$ which is mainly caused by the length of the identified PCPs. The longer length of PCPs in the second partition, in comparison with the first partition PCPs, allows BCframework to utilize its cheapest cost VM utilization strategy for the most numbers of operators of queries like Q2 and Q4. Whereas, the default scheduler assigns the cheapest and the most expensive VM type instances equally.

Table 8 shows that the BCframework reduces deadline violations of complex queries significantly. The deadline control mechanism of BCframework monitors the deadline violation of complex queries continuously whereas the default scheduler does not take into account the deadline of the queries.

Figure 12 shows that BCframework outperforms the default scheduler in $\mathrm{Vm}$ instance provisioning under 


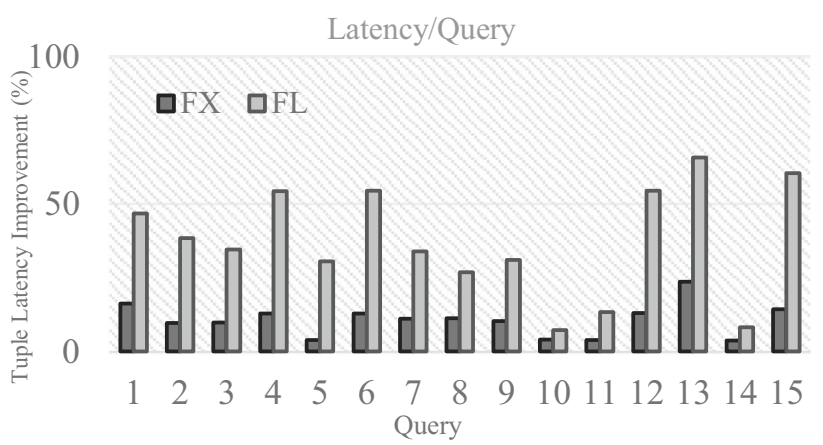

(a)

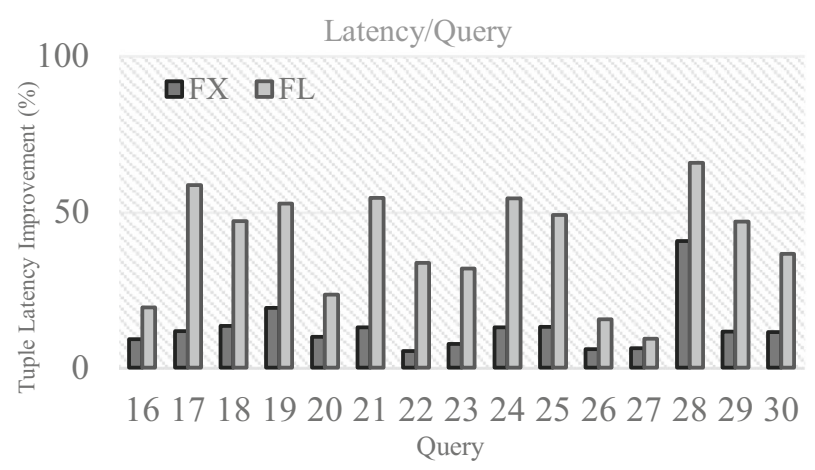

(b)

Fig. 10 Average tuple latency improvement under BigBench by BCframework. a Queries 1-15. b Queries 16-30

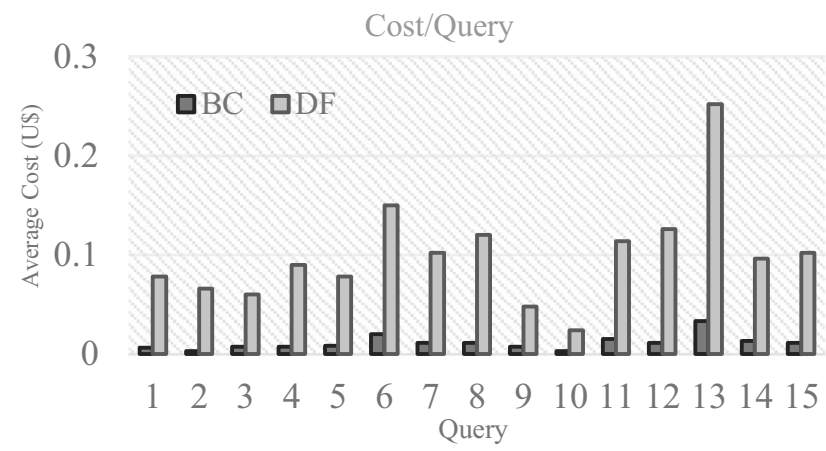

(a)

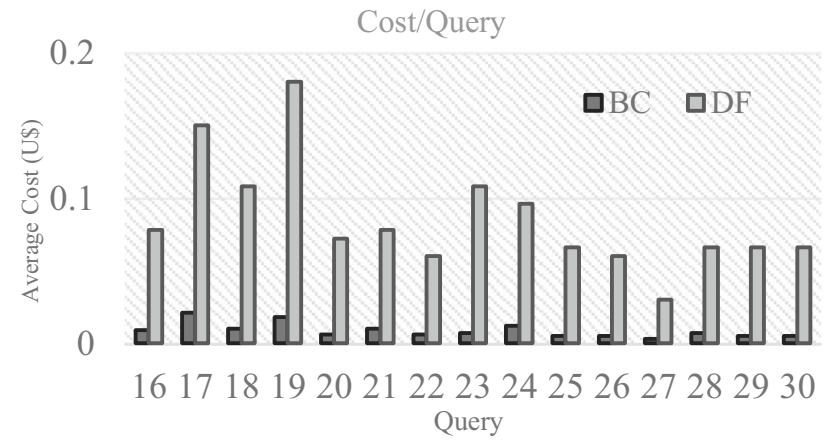

(b)

Fig. 11 The average cost (U\$) under BigBench. a Queries 1-15. b Queries 16-30
Table 8 Total number of deadline violations under BigBench queries

\begin{tabular}{|c|c|c|c|c|c|c|c|c|c|}
\hline$B s^{V e}$ & Q & $\alpha$ & $\mathrm{BC}$ & DF & $B s^{V e}$ & Q & $\alpha$ & $\mathrm{BC}$ & DF \\
\hline \multicolumn{10}{|c|}{ Queries 1-15 } \\
\hline FX & 1 & 2 & 1 & 12 & $\mathrm{FX}$ & 9 & 2 & 2 & 7 \\
\hline FL & & 4 & 0 & 10 & FL & & 3 & 6 & 10 \\
\hline $\mathrm{FX}$ & 2 & 2 & 2 & 12 & $\mathrm{FX}$ & 10 & 2 & 0 & 0 \\
\hline FL & & 3 & 5 & 5 & FL & & 3 & 0 & 0 \\
\hline $\mathrm{FX}$ & 3 & 2 & 2 & 9 & $\mathrm{FX}$ & 11 & 3 & 0 & 0 \\
\hline FL & & 3 & 4 & 10 & FL & & 3 & 5 & 7 \\
\hline $\mathrm{FX}$ & 4 & 2 & 3 & 11 & FX & 12 & 2 & 3 & 11 \\
\hline FL & & 3 & 3 & 15 & FL & & 3 & 4 & 14 \\
\hline $\mathrm{FX}$ & 5 & 3 & 0 & 0 & FX & 13 & 4 & 1 & 12 \\
\hline FL & & 3 & 4 & 10 & FL & & 4 & 3 & 15 \\
\hline FX & 6 & 2 & 0 & 9 & FX & 14 & 5 & 2 & 7 \\
\hline FL & & 3 & 4 & 15 & FL & & 6 & 3 & 8 \\
\hline FX & 7 & 2 & 3 & 14 & FX & 15 & 2 & 0 & 8 \\
\hline FL & & 3 & 4 & 10 & FL & & 3 & 5 & 15 \\
\hline $\mathrm{FX}$ & 8 & 2 & 1 & 6 & FX & 16 & 5 & 3 & 13 \\
\hline FL & & 3 & 3 & 12 & FL & & 6 & 5 & 11 \\
\hline \multicolumn{10}{|c|}{ (b) Queries 16-30 } \\
\hline $\mathrm{FX}$ & 17 & 5 & 3 & 12 & FX & 25 & 5 & 1 & 11 \\
\hline FL & & 6 & 3 & 13 & FL & & 6 & 3 & 13 \\
\hline $\mathrm{FX}$ & 18 & 2 & 3 & 13 & FX & 26 & 2 & 3 & 12 \\
\hline FL & & 3 & 4 & 14 & FL & & 3 & 5 & 10 \\
\hline $\mathrm{FX}$ & 19 & 2 & 2 & 11 & FX & 27 & 2 & 0 & 0 \\
\hline FL & & 3 & 1 & 11 & FL & & 3 & 0 & 0 \\
\hline FX & 20 & 2 & 2 & 11 & FX & 28 & 2 & 0 & 0 \\
\hline FL & & 3 & 6 & 10 & FL & & 3 & 0 & 0 \\
\hline $\mathrm{FX}$ & 21 & 2 & 5 & 15 & FX & 29 & 2 & 3 & 10 \\
\hline FL & & 3 & 8 & 15 & FL & & 3 & 6 & 11 \\
\hline $\mathrm{FX}$ & 22 & 2 & 6 & 11 & FX & 30 & 2 & 3 & 10 \\
\hline FL & & 3 & 6 & 11 & FL & & 3 & 6 & 10 \\
\hline $\mathrm{FX}$ & 23 & 2 & 3 & 8 & & & & & \\
\hline FL & & 3 & 3 & 9 & & & & & \\
\hline $\mathrm{FX}$ & 24 & 2 & 0 & 10 & & & & & \\
\hline FL & & 3 & 3 & 15 & & & & & \\
\hline
\end{tabular}

complex queries. The default scheduler assigns each operator of a query to a $\mathrm{Vm}$ instance while BCframework provisions $\mathrm{Vm}$ instances by considering structure of the queries and applies the different strategies for a query. For query Q11, BCframework has identified six PCPs and six instances are provisioned while the default scheduler assigns the provisioned eighteen instances to the nineteen operators. For query Q13, both BCframework and the default scheduler assign the same number of $V m$ instances because BCframework provisions eighteen $\mathrm{Vm}$ instances for eighteen identified PCPs and the default scheduler 


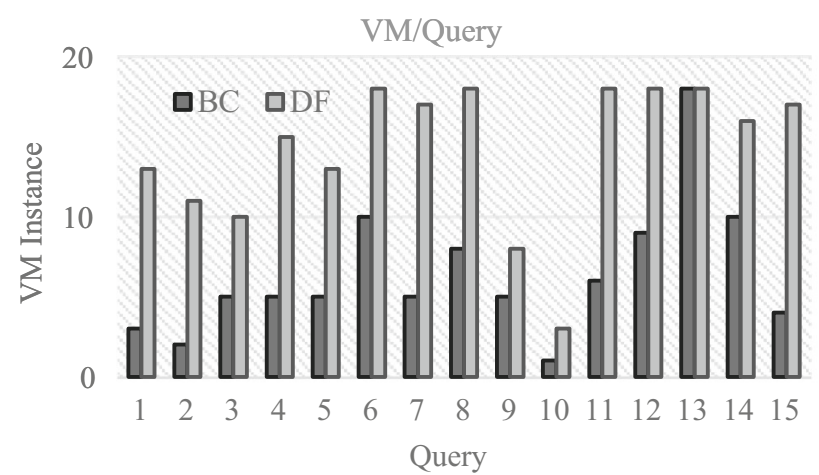

(a)

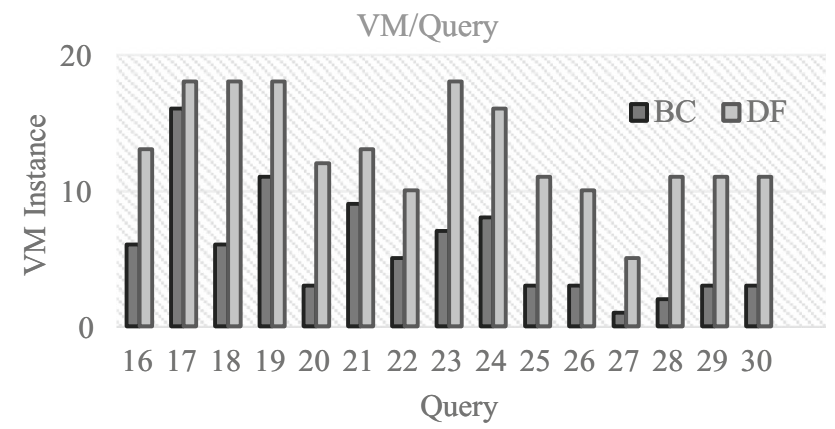

(b)

Fig. 12 The number of provisioned $V m$ instances under BigBench queries. a Queries 1-15. b Queries 16-30

distributes forty-two operators of the query to all eighteen provisioned instances.

\subsection{Real-world queries}

As shown in Fig. 13a, BCframework reduces the average tuple latency of WordCount and Top_N queries up to $36 \%$ and $41 \%$ respectively. The state-of-the-art scheduler prioritizes WordCount's operators according to their position in the query to order the operators with highest computing time first. Furthermore, the scheduler's policy for scheduling the operators on virtual machines is based on the minimum total time of available time and computing time which leads to schedule the operators to three $\mathrm{Vm}$ instances and increase the traffic load between these instances. Whereas, BCframework partitions WordCount's operators based on the $B f$ size and schedules them on two $V m$ instances based on the available time, computing power and computing cost of the operators which reduce the traffic load of virtual machines. To schedule Top_N, the

Fig. 13 Performance evaluation of BCframework and the state-ofthe-art scheduler under WordCount and Top_N queries. a Average tuple latency improvement b Average cost. c Total number of deadline violations. d The number of provisioned $\mathrm{Vm}$ instances

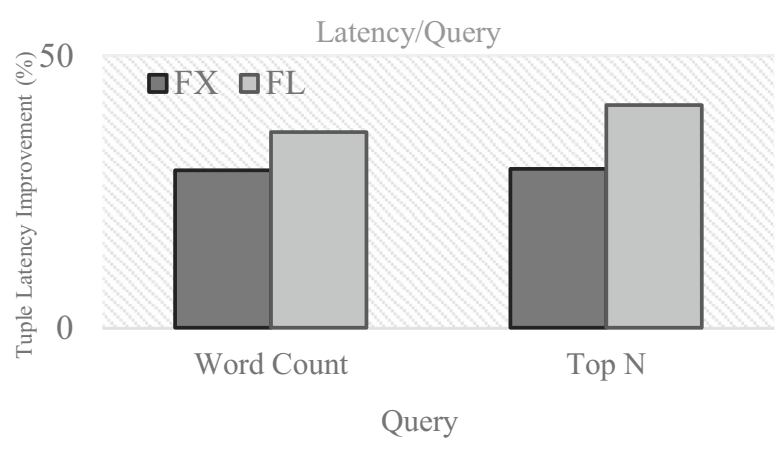

(a)

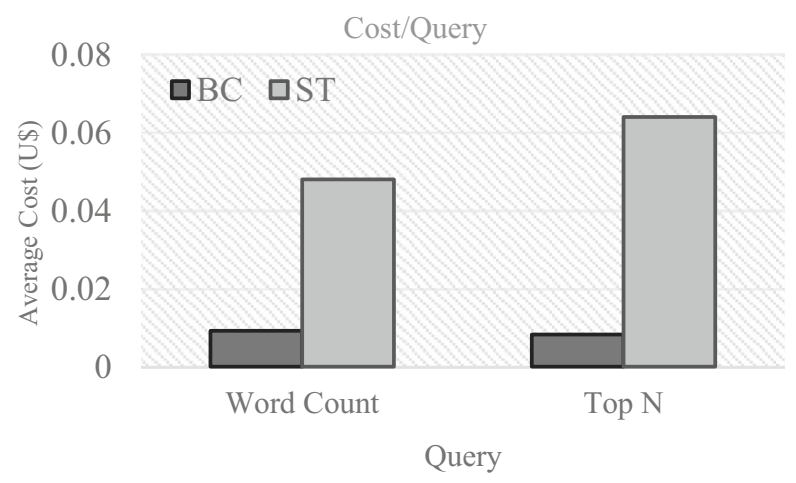

(b)

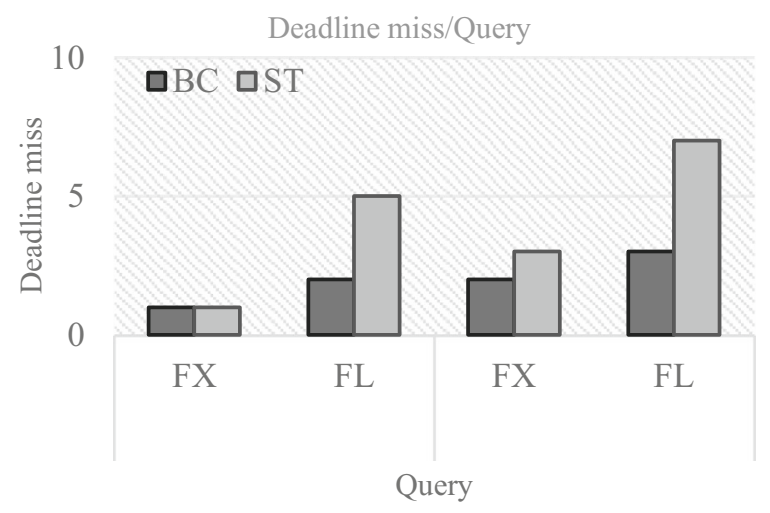

(c)

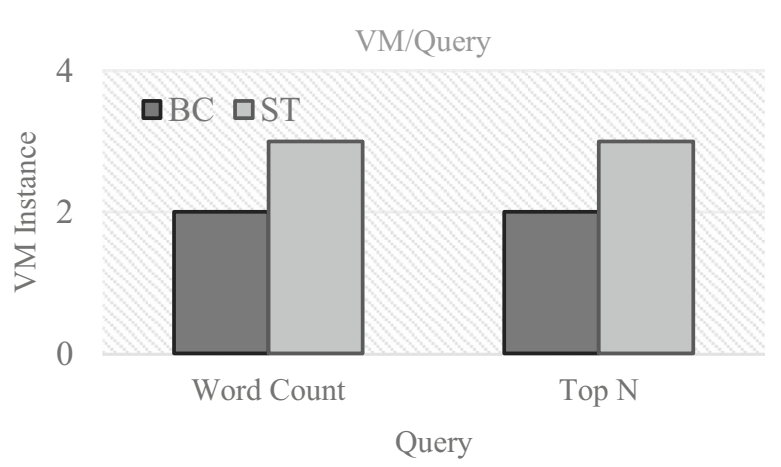

(d) 
state-of-the-art scheduler also considers three $V m$ instances while BCframework only considers two instances.

Figure 13b shows the cost reduction of the WordCount and Top_N queries. For these queries, the state-of-the-art scheduler takes into account virtual machines whose computing time is minimized which leads to an increase of utilization cost of the provisioned $V m$ instances while BCframework considers the highest computing time for first partition operators and the lowest computing utilization cost for the second partition operators which leads to reduce the computation utilization cost of a query.

As shown in Fig. 13c, in the face of fixed and fluctuating scenarios, BCframework either completely eliminates deadline violations of the WordCount and Top_N queries or reduces them significantly. The BCframework's deadline control mechanism monitors the deadline violation of the queries continuously and reschedules operators using a rescheduling strategy which provisions additional Vm instances. Whereas the state-of-the-art scheduler applies its primary scheduling strategy to reschedule the operators of a query.

Figure 13d shows that BCframework outperforms the state-of-the-art scheduler in $\mathrm{Vm}$ instance provisioning under real-world queries. The state-of-the-art scheduler's policy for provisioning $\mathrm{Vm}$ instances is based on minimum total time of available time and computing time of $\mathrm{Vm}$ instances which leads to the provision of three $\mathrm{Vm}$ instances Whereas, BCframework partitions WordCount's operators based on the $B f$ size and schedule them on two virtual machines based on the available time, computing power and computing utilization cost of the operators. To provision $V m$ instances for Top_N, the state-of-the-art scheduler also choices three virtual machine instances while BCframework just considers two instances.

\subsection{Parameter sensitivity}

To determine the parameters Threshold and $\alpha$, two group of experiments has conducted. For the first experiments, the deadline misses of the queries are evaluated to determine the deadline factor $\alpha$ and for the second experiments, the deadline misses and utilization costs of the queries are evaluated to determine the Threshold parameter. For both experiments, the queries are executed using a range of different values for $\alpha$ and Threshold while respecting the fixed scenario $B s^{V e}=1000$ tuples/s, and fluctuated scenario $B s^{V e}=15,000$ tuples/s. Figure 14 illustrates the result of the parameter sensitivity of the simple query Q1 of Linear Road Benchmark and complex query Q1 of BigBench.

As shown in Fig. 14a and b for the simple query Q1 and complex query Q1 under fix and fluctuating scenarios, the

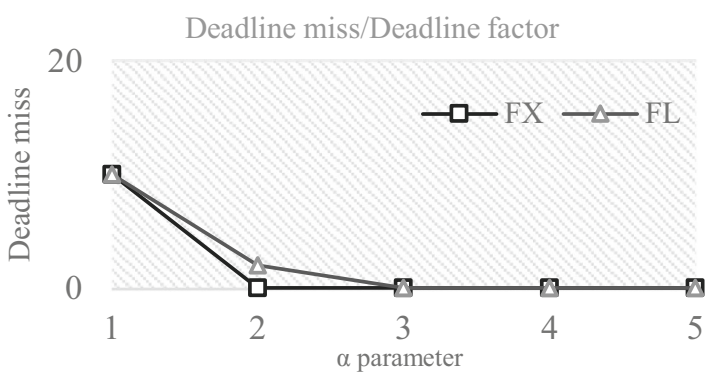

(a)

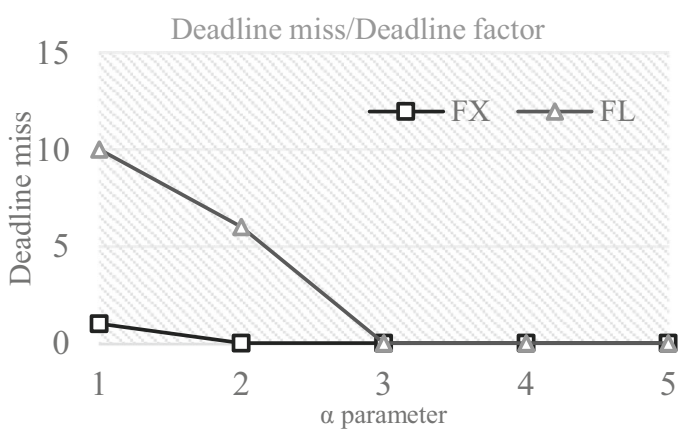

(b)

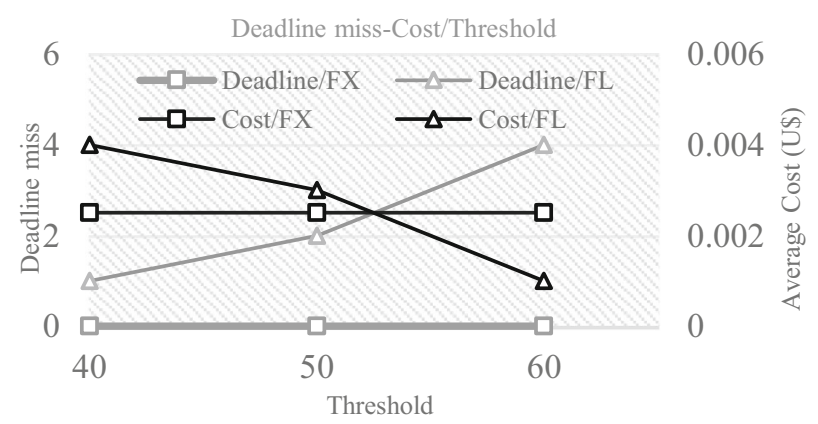

(c)

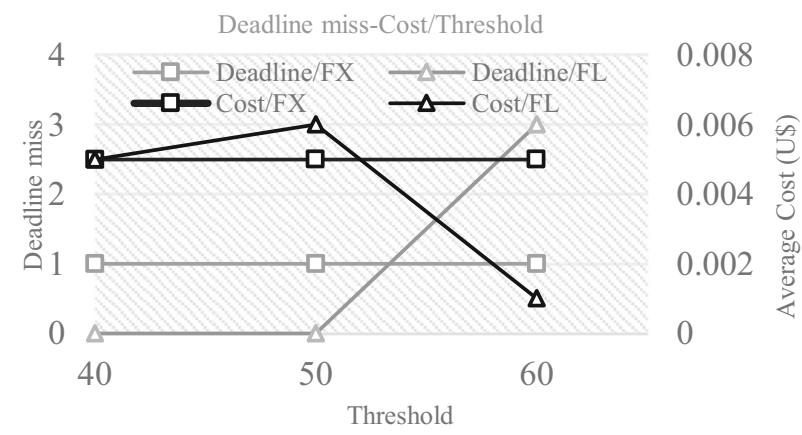

(d)

Fig. 14 Evaluation of BCframework's parameter sensitivity. a Effect of $\alpha$ on the deadline miss under simple query Q1. b Effect of $\alpha$ on the deadline miss under complex query Q1. c Effect of Threshold on the deadline miss and the utilization cost under the simple query Q1. d Effect of the Threshold on the deadline miss and the utilization cost under the complex query Q1 
deadline miss will be increase by decreasing deadline factor $\alpha$. For the query Q1 under both scenarios, by the most restricted value of $\alpha$, e.g., $\alpha=2$, BCframework cannot guarantee the execution of the query before its deadline. Whereas, for a relaxed value of $\alpha$, e.g., $\alpha=4$, the query can be executed without deadline violation. Therefore, for the simple query Q1, the $\alpha$ parameter is adjusted to 3. In a similar way, for the complex query Q1, the $\alpha$ parameter is adjusted to two and four for fixed and fluctuated scenarios respectively. The adjusted value of other $\alpha$ parameters of all simple and complex queries are illustrated in Tables 7 and 8 respectively.

As shown in Fig. 14c and d for the simple query Q1 and complex query Q1 under fix and fluctuating scenarios, decreasing the value of Threshold leads to a decrease of the deadline violations and to an increase of utilization cost of the query. By decreasing the Threshold value, deadline controller of BCframework has to react more quickly and must provision more $V M$ instances to decrease the deadline violations that result in increasing the utilization cost. On the other hand, by increasing Threshold value, deadline controller just monitors the execution of the queries.

\subsection{Discussion}

According to the conducted experiments and their analysis, we can conclude that BCframework is able to correctly partition the streaming Big Data analytic queries based on its input Big Data stream. Experimental results show that BCframework is able to correctly partition simple and complex analytic queries of Big Data stream.

Furthermore, BCframework outperforms the Storm default scheduler in the average tuple latency reduction under the simple and complex streaming Big Data analytic queries up to $29.6 \%$ and $65.8 \%$ respectively. In addition, BCframework outperforms the state-of-the-art scheduler in the average tuple latency reduction under the real-world queries up to $41 \%$. Experimental result have shown that BCframework is able to reduce the average tuple latency of simple queries mainly through the appropriate scheduling of virtual machines. For complex queries, BCframework can also be used to efficiently reduce the average tuple latency through the efficient scheduling and provisioning of VM instances.

In addition, BCframework outperforms Storm default scheduler in the reduction of public Cloud resource utilization cost and the deadline violations of streaming Big Data analytic queries. Experimental results have proven that BCframework can reduce the utilization cost of the simple and complex Big Data stream analytical queries through the appropriate VM utilization policy for the operators of the query. Furthermore, experiments have shown that BCframework reduces the deadline violation using the deadline monitoring mechanism and efficient VM provisioning policy.

Finally, BCframework performs best in complex fluctuating streaming Big Data analytic queries. Experimental results prove that most BCframework optimization techniques are completely utilized by queries with complex structures and fluctuating input Big Data streams.

\section{Conclusion}

The demand for streaming Big Data analysis applications is increasing. As the demand is growing, the efficient execution of the applications using suitable Cloud platforms become more important. However, current platform schedulers are not suitable for these applications in an effective manner. This study presents BCframework, a framework designed to efficiently schedule the accepted streaming Big Data analytic queries in the public Cloud. The BCframework proposes partitioning, critical path determination, scheduling and provisioning algorithms for the original and replicated operators. BCframework is developed based on the idea of using more than one scheduling strategy for streaming Big Data analytic query original operator and replicated operator which is used by the proposed algorithms to reduce the utilization cost, the deadline miss ratio of queries and mitigate public Cloud performance fluctuation. BCframework partitions a query based on its Big Data stream characteristics and applies a set of efficient provisioning and scheduling strategies for each partition during the execution of query.

Experimental results show that, compared to Storm's default scheduler and the state-of-the-art scheduler, BCframework is able to efficiently reduce tuple latency of streaming Big Data analytic queries up to $65 \%$ and $41 \%$ respectively. Furthermore, BCframework significantly decreases the likelihood of the deadline misses and utilization cost of streaming Big Data analytic queries.

In the future work, it is planned to extend BCframework on multiple cloud resources. A more efficient resource provisioning mechanism using streaming Big Data fluctuation prediction model will be considered in our following work. Moreover, experiments that execute Health Big Data queries will also be included.

Open Access This article is distributed under the terms of the Creative Commons Attribution 4.0 International License (http://creative commons.org/licenses/by/4.0/), which permits unrestricted use, distribution, and reproduction in any medium, provided you give appropriate credit to the original author(s) and the source, provide a link to the Creative Commons license, and indicate if changes were made. 


\section{References}

1. Chen, M., Mao, S., Zhang, Y., Leung, V.C.M.: Big Data: Related Technologies, Challenges and Future Prospects. Springer, New York (2014)

2. Safaei, A.A.: Real-time processing of streaming big data. RealTime Syst. 53(1), 1-44 (2017)

3. Barlow, M.: Real-Time Big Data Analytics Emerging Architecture. O'Reilly Media, Inc, Newton (2013)

4. Berman, J.J.: Principles of Big Data: Preparing, Sharing, and Analyzing Complex Information. Elsevier, Amsterdam (2013)

5. Pedrycz, W., Chen, S.-M.: Information Granularity, Big Data, and Computational Intelligence. Springer, New York (2014)

6. Jackson, K.R., Ramakrishnan, L., Muriki, K., Canon, S., Cholia, S., Shalf, J., et al. (eds.): Performance analysis of high performance computing applications on the amazon web services cloud. In: Proceedings of the 2010 IEEE Second International Conference on Cloud Computing Technology and Science (CloudCom), IEEE (2010)

7. Apache Storm [Online]. http://storm.apache.org/index.html. Accessed 4 Feb 2016

8. Xu, J., Chen, Z., Tang, J., Su, S., (eds.): T-storm: Traffic-aware online scheduling in storm. In: Proceedings of the 2014 IEEE 34th International Conference on Distributed Computing Systems (ICDCS), IEEE (2014)

9. Cardellini, V., Grassi, V., Lo Presti, F., Nardelli M., (eds.): Distributed QoS-aware scheduling in Storm. In: Proceedings of the 9th ACM International Conference on Distributed EventBased Systems, ACM (2015)

10. Li, T., Tang, J., Xu, J., (eds.): A predictive scheduling framework for fast and distributed stream data processing. In: Proceedings of the 2015 IEEE International Conference on Big Data (Big Data), IEEE (2015)

11. Cardellini, V., Grassi, V., Lo Presti, F., Nardelli, M.: Optimal operator replication and placement for distributed stream processing systems. ACM SIGMETRICS Perf. Eval. Rev. 44(4), 11-22 (2017)

12. Cardellini, V., Grassi, V., Lo Presti, F., Nardelli, M., (eds.): Optimal operator placement for distributed stream processing applications. In: Proceedings of the 10th ACM International Conference on Distributed and Event-based Systems, ACM (2016)

13. De Matteis, T., Mencagli, G., (eds.): Keep calm and react with foresight: Strategies for low-latency and energy-efficient elastic data stream processing. In: Proceedings of the 21st ACM SIGPLAN Symposium on Principles and Practice of Parallel Programming, ACM (2016)

14. Dwarakanathan, S, (ed.): S-Flink: schedule for QoS in Flink Using SDN. In: Proceedings of the IEEE 40th Annual Computer Software and Applications Conference (COMPSAC), 2016, IEEE (2016)

15. Kulkarni, S., Bhagat, N., Fu, M., Kedigehalli, V., Kellogg, C., Mittal, S., et al. (eds.): Twitter heron: stream processing at scale. In: Proceedings of the 2015 ACM SIGMOD International Conference on Management of Data, ACM (2015)

16. Abadi, D.J., Carney, D., Çetintemel, U., Cherniack, M., Convey, C., Lee, S., et al.: Aurora a new model and architecture for data stream management. VLDB J. 12(2), 120-139 (2003)

17. Gulisano, V., Jimenez-Peris, R., Patino-Martinez, M., Soriente, C., Valduriez, P.: Streamcloud: an elastic and scalable data streaming system. IEEE Trans. Parallel Distrib. Syst. 23(12), 2351-2365 (2012)

18. Li, C., Zhang, J., Luo, Y.: Real-time scheduling based on optimized topology and communication traffic in distributed real- time computation platform of storm. J. Netw. Comput. Appl. 87, 100-115 (2017)

19. Sun, D., Zhang, G., Yang, S., Zheng, W., Khan, S.U., Li, K.: ReStream: real-time and energy-efficient resource scheduling in big data stream computing environments. Inf. Sci. 319, 92-112 (2015)

20. Sun, D., Zhang, G., Wu, C., Li, K., Zheng, W.: Building a fault tolerant framework with deadline guarantee in big data stream computing environments. J. Comput. Syst. Sci. 89, 4-23 (2017)

21. Kaur, N., Sood, S.K.: Dynamic resource allocation for big data streams based on data characteristics (5Vs). Int. J. Netw. Manag. 27, e1978 (2017)

22. Sun, D., Huang, R.: A stable online scheduling strategy for realtime stream computing over fluctuating big data streams. IEEE Access 4, 8593-8607 (2016)

23. Kaur, N., Sood, S.K.: Efficient resource management system based on 4Vs of big data streams. Big Data Res. (2017). https:// doi.org/10.1016/j.bdr.2017.02.002

24. Huang, R., Sun, D.: Analysing and evaluating topology structure of online application in Big Data stream computing environment. Int. J. Wireless Mobile Comput. 10(4), 317-324 (2016)

25. Tolosana-Calasanz, R., Bañares, J.Á., Pham, C., Rana, O.F.: Resource management for bursty streams on multi-tenancy cloud environments. Future Gener. Comput. Syst. 55, 444-459 (2016)

26. Rahman, M.M., Graham, P.: Responsive and efficient provisioning for multimedia applications. Comput. Electr. Eng. 53, 458-468 (2016)

27. Zhang, Q., Chen, Z., Yang, L.T.: A nodes scheduling model based on Markov chain prediction for big streaming data analysis. Int. J. Commun. Syst. 28(9), 1610-1619 (2015)

28. Peng, J-j, Zhi, X-f, Xie, X-1: Application type based resource allocation strategy in cloud environment. Microprocess. Microsyst. 47, 385-391 (2016)

29. Baughman, A.K., Bogdany, R.J., McAvoy, C., Locke, R., O'Connell, B., Upton, C.: Predictive cloud computing with big data: professional golf and tennis forecasting [application notes]. IEEE Comput. Intell. Mag. 10(3), 62-76 (2015)

30. Sun, D., et al.: Rethinking elastic online scheduling of big data streaming applications over high-velocity continuous data streams. J. Supercomput. 74(2), 615-636 (2018)

31. Amazon AWS EC2 pricing model [Online]. www.amazon.com/ EC2/pricing/pricingmodel.html. Accessed 7 June 2016

32. Fegaras, L.: Incremental query processing on Big Data streams. IEEE Trans. Knowl. Data Eng. 28(11), 2998-3012 (2016)

33. Byun, E.-K., Kee, Y.-S., Kim, J.-S., Maeng, S.: Cost optimized provisioning of elastic resources for application workflows. Future Gener. Comput. Syst. 27(8), 1011-1026 (2011)

34. Shi, J., et al.: Elastic resource provisioning for scientific workflow scheduling in cloud under budget and deadline constraints. Clust. Comput. 19(1), 167-182 (2016)

35. Amazon EC2 [Online]. https://aws.amazon.com/ec2/. Accessed 10 May 2016

36. Apache Spark - Unified Analytics Engine for Big Data [Online]. https://spark.apache.org/ Accessed 10 Apr 2018

37. Ghazal, A., Rabl, T., Hu, M., Raab, F., Poess, M., Crolotte, A., et al. (eds.): BigBench: towards an industry standard benchmark for big data analytics. In: Proceedings of the 2013 ACM SIGMOD international conference on Management of data, ACM (2013)

38. Arasu, A., Cherniack, M., Galvez, E., Maier, D., Maskey, A.S., Ryvkina, E., et al. (eds.): Linear road: a stream data management benchmark. In: Proceedings of the Thirtieth international conference on Very large data bases, vol. 30, VLDB Endowment (2004)

39. Tutorial [Online]. http://storm.apache.org/releases/1.1.2/Tutorial. html. Accessed 10 Apr 2018 
40. Common Toplogy Patterns [Online]. http://storm.apache.org/ releases/1.2.1/Common-patterns.html. Accessed 10 Apr 2018

41. Byrne, J., et al.: A review of cloud computing simulation platforms and related environments. In: Proceedings of the 7th International Conference on Cloud Computing and Services Science. SCITEPRESS-Science and Technology Publications, Lda Portugal (2017)

42. Rabl, T., et al.: BigBench Specification V0. 1, in Specifying Big Data Benchmarks, pp. 164-201. Springer, New York pp (2014)

43. Higashino, W.A., Capretz, M.A., Bittencourt, L.F.: CEPSim: modelling and simulation of Complex Event Processing systems in cloud environments. Fut. Gener. Comput. Syst. 65, 122-139 (2016)

44. Calheiros, R.N., et al.: CloudSim: a toolkit for modeling and simulation of cloud computing environments and evaluation of resource provisioning algorithms. Software 41(1), 23-50 (2011)

45. CEPSim - Simulator for CEP/SP systems. https://github.com/vir sox/cepsim. Accessed 10 Apr 2018

46. Han, R., Lu, X., Xu, J.: On Big Data benchmarking. In: Zhan, J., Han, R., Weng, C. (eds.) Big Data Benchmarks, Performance Optimization, and Emerging Hardware. BPOE 2014. Lecture Notes in Computer Science. Springer, Cham (2014)

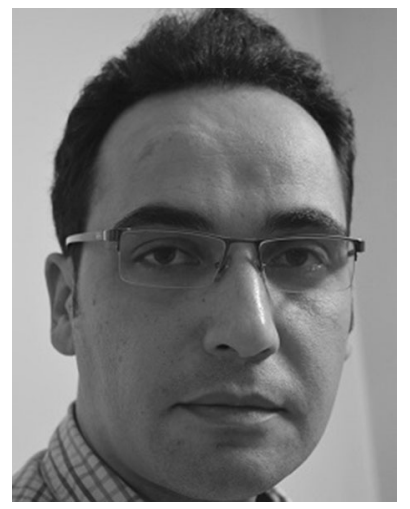

Mahmood Mortazavi Dehkordi obtained his M.Sc. in software engineering from University of Isfahan. He is faculty member of Sheikhbahaee University. Presently he is pursuing his Ph.D. in Big Data and cloud computing at University of Isfahan. His research areas are Big Data, Cloud Computing and Software Engineering.

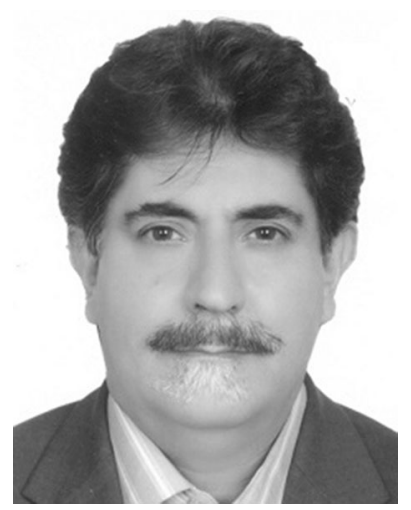

Kamran zamanifar received his B.Sc. and M.Sc. from the University of Tahran in electronic engineering. He got his $\mathrm{Ph} . \mathrm{D}$. in parallel and distributed systems form University of Leeds in UK. He is associate professor at the software engineering department at university of Isfahan. His research interests are in parallel and distributed computing, cloud computing, soft computing and pervasive computing.

Publisher's Note Springer Nature remains neutral with regard to jurisdictional claims in published maps and institutional affiliations. 